Review

\title{
An Overview of Acetic Acid Ulcer Models — The History and State of the Art of Peptic Ulcer Research-
}

\author{
Susumu OKABE*,1) and Kikuko Amagase \\ Department of Applied Pharmacology, Kyoto Pharmaceutical University; Misasagi, Yamashina, Kyoto 602-0897, Japan.
} Received April 28, 2005

\begin{abstract}
Four types of experimental chronic ulcer models, named acetic acid ulcer models, have been developed to examine the healing process of peptic ulcers, screen anti-ulcer drugs, and better evaluate the adverse effects of various anti-inflammatory drugs on the gastrointestinal mucosa. The model easily and reliably produces round, deep ulcers in the stomach and duodenum, allowing acetic acid ulcer production in mice, rats, Mongolian gerbils, guinea pigs, cats, dogs, miniature pigs, and monkeys. These ulcer models highly resemble human ulcers in terms of both pathological features and healing process. The models have been established over the past 35 years and are now used throughout the world by basic and clinical scientists. One of the characteristic features of acetic acid ulcers in rats is the spontaneous relapse of healed ulcers $>100 \mathrm{~d}$ after ulceration, an endoscopically confirmed phenomenon. Indomethacin significantly delays the healing of acetic acid ulcers, probably by reducing endogenous prostaglandins and inhibiting angiogenesis in ulcerated tissue. Helicobacter pylori significantly delays healing of acetic acid ulcers and causes relapse of healed ulcers at a high incidence in Mongolian gerbils. Anti-secretory drugs (e.g. omeprazole), prostaglandin analogs, mucosal defense agents (e.g. sucralfate), and various growth factors all significantly enhance healing of acetic acid ulcers. Gene therapy with epidermal growth factor and vascular endothelial growth factor applied to the base of acetic acid ulcers in rats is effective in enhancing ulcer healing. Since an inhibitor of nitric oxide syntase prevents ulcer healing, nitric oxide might be involved in the mechanism underlying ulcer healing. We conclude that acetic acid ulcer models are quite useful for various studies related to peptic ulcers.
\end{abstract}

Key words experimental chronic ulcer; ulcer healing; anti-ulcer drug; Helicobacter pylori; gene therapy

\section{INTRODUCTION}

"The most important undertaking in science is the establishment of experimental methods" - Ivan P. Pavlov (18491936). Pavlov's statement is readily applicable to ulcer research, i.e., establishing an experimental ulcer model that resembles human ulcers is of utmost importance. Nonetheless, although easily stated, developing an experimental method can prove difficult, especially when such a method lies beyond the frontiers of contemporary science. This review article describes the process underlying establishment of one such experimental method, namely how a chronic ulcer model, currently called the "acetic acid ulcer" model, was established in laboratory animals. Since its development in 1969 , the acetic acid ulcer model has become well established in the scientific community. The number of published studies in which acetic acid ulcer models have been utilized numbers approximately $30-45$ per year over the past decade. The reasons underlying the model's frequent use as the chronic ulcer model of choice appear to be as follows. 1) The ulcer induction procedure is quite simple, readily resulting in ulcers of consistent size and severity at an incidence of $100 \%$. 2) The ulcer models highly resemble human ulcers in terms of both pathological features and healing mechanisms. Indeed, spontaneous relapse of healed ulcers is frequently observed, just as in peptic ulcer patients. 3) The ulcers respond well to various anti-ulcer drugs, such as acid pump inhibitors, sucralfate, and several growth factors. Moreover, both steroidal and non-steroidal anti-inflammatory drugs negatively impact healing of the experimental ulcers. The ulcer models are now used as the standard model for screening compounds as potential anti-ulcer drugs. The below summarizes the history of the development, as well as the utility of acetic acid ulcer models. In addition, state of the art research concerning the mechanisms underlying the healing of acetic acid ulcers is overviewed.

\section{ACETIC ACID ULCER MODELS}

2.1. Type 1 Ulcer Model In the early $1960 \mathrm{~s}$, there were only a few acute gastric ulcer models routinely used in the laboratory, such as pylorus-ligation ulcers (Shay ulcers) and stress-induced ulcers. ${ }^{2,3)}$ From a pathological point of view, however, these ulcer models were inappropriate for the study of human gastric ulcers, which penetrate the muscular layer of the glandular area and occasionally relapse after healing. In an attempt to induce such ulcers in rats, repetitive stressful conditions, such as cold exposure, water immersion, or electric shock were utilized to attempt to induce penetrating ulcers, but remained unsuccessful. $\left.{ }^{4}\right)$

During an autopsy for an unrelated experiment, I (SO) serendipitously discovered that the stomach of a rat had developed a round, deeply penetrating gastric ulcer (ID approximately $2 \mathrm{~mm}$ ). As it is common knowledge that rats do not spontaneously develop ulcers, it was postulated that the ulcer might have been induced by accidental direct injection of a compound that was intended for injection into the intraperitoneal cavity, but was, instead, mistakenly introduced into the gastric wall. Since the accidentally injected compound was not a readily available one, I experimentally injected into the stomach wall various known necrotizing agents, such as aspirin, cortisone, carragennin, ethanol, and hydrochloric acid, in an attempt to reproduce a similar type of ulcer. Unfortunately, such compounds were not successful, only inducing superficial damage, similar to the appearance of stress-induced ulcers. 
Submucosal injection (1)

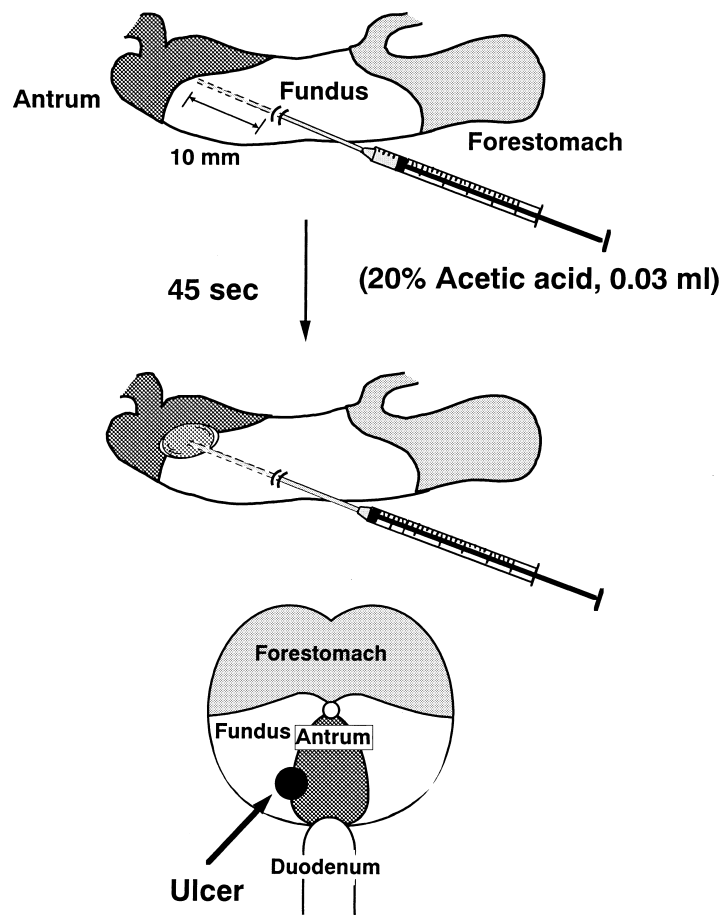

\section{Serosal application (2)}
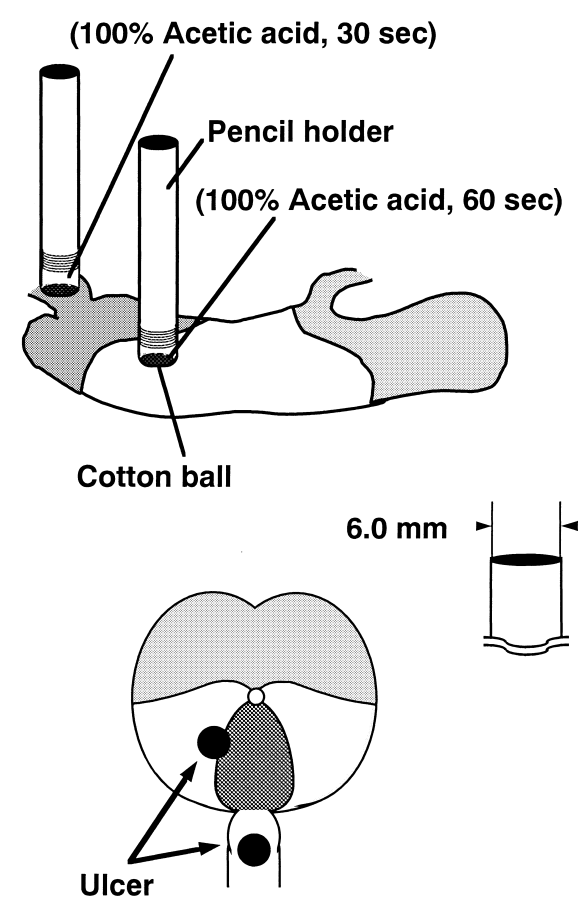

Fig. 1. Schematic Drawing of the Experimental Procedure for Producing Acetic Acid Ulcers in Small Animals

(1) Submucosal injection of acetic acid solution into the rat stomach (Type 1 ulcer). (2) Local application of acetic acid solution to the serosa of either the stomach or duodenum (Type 2 ulcer).

At the time of these experiments, colleagues in our laboratory were screening anti-inflammatory drugs by injecting an acetic acid solution into rat paws. Professor Keijiro Takagi, my supervisor at the time, suggested that instead of the above-mentioned agents, injection of an acetic acid solution into the gastric mucosal layer might allow consistent induction of penetrating ulcers. To our great surprise, it was found that such an acid solution successfully necrotized the mucosal layer, producing deep, round/oval ulcers similar to the ulcer observed in the initial discovery. Our goal of producing chronic gastric ulcers in rat stomachs suddenly appeared much more readily attainable.

A standard procedure was established, which is described as follows. The procedure begins with laparotomy of etheranesthetized rats via midline epigastric incision. After exposing the stomach, $20 \%$ acetic acid $(0.02 \mathrm{ml})$, is injected into the submucosal layer of the glandular portion of the anterior wall (Fig. 1) with a microsyringe $(0.05 \mathrm{ml})$. We initially reported that the location of the injected acetic acid solution was the subserosal area. Nonetheless, I subsequently confirmed that the location of the acid injection is actually the submucosal area. At the time of injection, a thumb is placed tightly on the inserted needle so as to avoid solution leakage. After injection, the needle is withdrawn, but the thumb is maintained at the injection site for $45 \mathrm{~s}$ to prevent acetic acid leakage upon removal of the needle. Accuracy of the injection is easily confirmed by observation of wheal-like swelling at the injection site. The operation requires approximately 4-5 min per animal. After closing the abdomen, the animals are routinely maintained. Thereafter, the animals are sacrificed at regular intervals to assess ulcer healing. Within
30 min after injection, the gastric mucosal surface was damaged (Fig. 2). Five days after acid injection, round/oval, deep ulcers (approximately $50-60 \mathrm{~mm}^{2}$ ) are observed in the antrum or in the area near the junction of the antrum and corpus corresponding to the injected area (Fig. 3). We consequently designated the 5th day after acid injection as the first day of ulceration. Histological sections confirm the presence of an ulcer penetrating the entire gastric wall; i.e., muscle tissue is completely absent in the area of ulceration (Fig. 4). Leukocyte exudate, edema, and cellular infiltration are frequently observed in the submucosal layer around the ulcer edge.

The manuscript that described the details of this new method for ulcer induction (now generally known as Type 1 acetic acid ulcers) was subsequently published. ${ }^{5)}$ At that time, however, most gastric ulcer research focused on issues associated with ulcer pathogenesis, rather than ulcer healing. Despite the simplicity and reproducibility, researchers were not interested in either producing penetrating ulcers by this new method or studying the mechanisms of ulcer healing. Accordingly, this first paper was given little attention for over a decade. One of the reasons that the paper received little notice appears to be that the method of ulceration is a physicochemical method, as opposed to a physiologic model. Accordingly, most researchers originally dismissed this model since they deemed it inappropriate for the study of ulcer pathogenesis. Nonetheless, researchers gradually began to recognize the potential of the method, as there was no alternative way to both study the healing process of ulcers and screen anti-ulcer drugs. Notably, acetic acid ulcers produced with this model persist for more than $250 \mathrm{~d}$ without interven- 

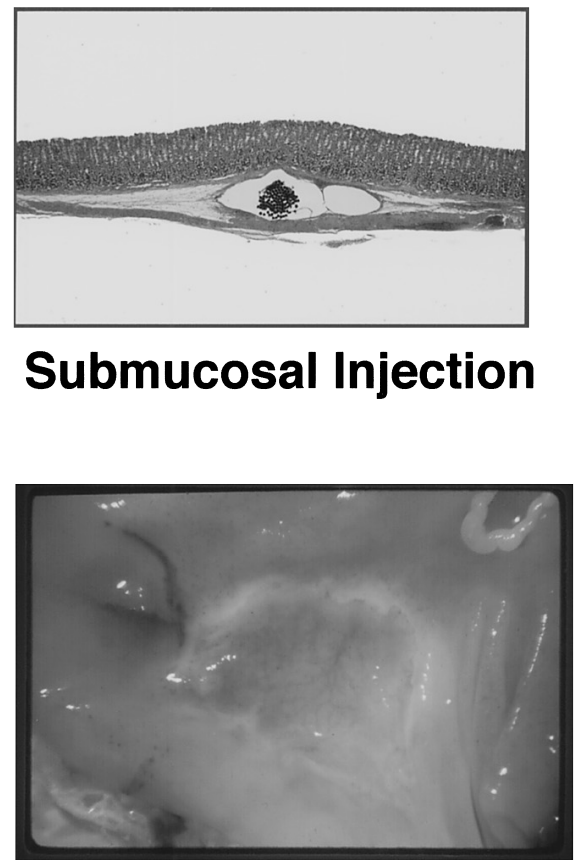

$10 \mathrm{~min}$

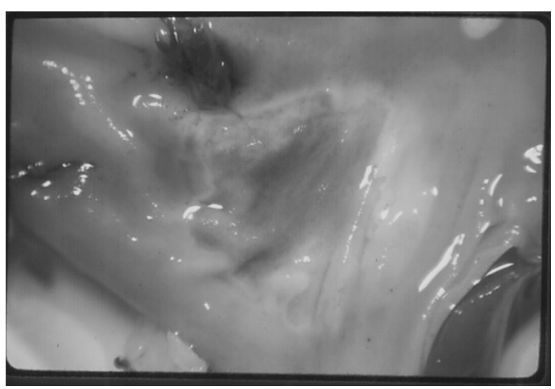

$5 \min$

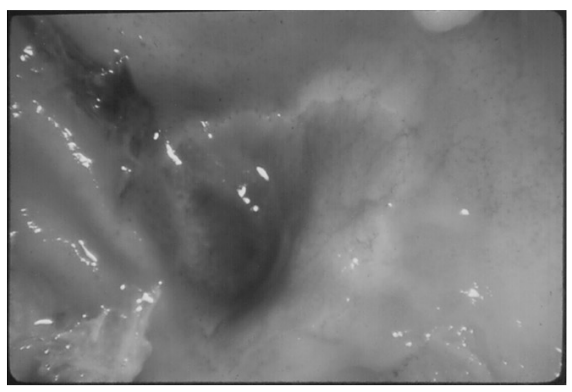

$30 \mathrm{~min}$

Fig. 2. Microscopic Appearance of Rat Gastric Wall at the Site of Acetic Acid Solution Injection

Gross appearance of the gastric mucosa 5, 10, and 30 min after acetic acid injection. To demonstrate the site of injection, a thread (group of dotted black points) was injected into the gastric wall instead of acetic acid solution; published figure in Yakugaku Zasshi, volume 125, pp. 21 (2005).

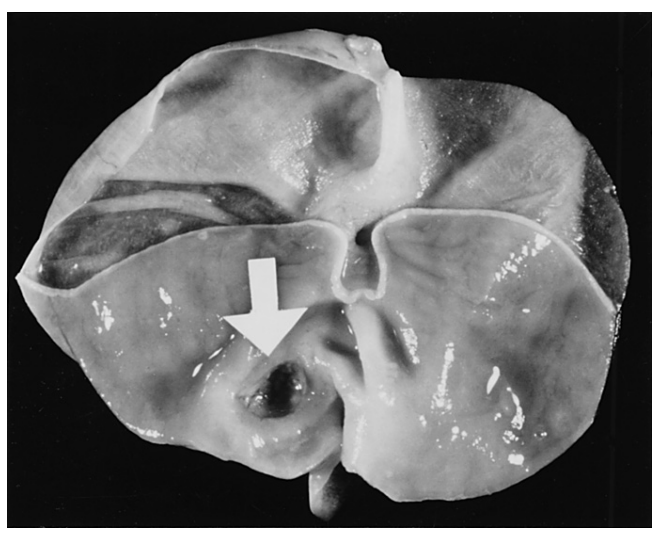

Fig. 3. Gross Appearance of Acetic Acid Ulcer (Type 1)

A round, deep ulcer was produced in the rat stomach $5 \mathrm{~d}$ after acid injection; published figure in Yakugaku Zasshi volume 125, pp. 19 (2005).

tion. ${ }^{6)}$ Furthermore, our studies demonstrated that 1.5 years following ulceration, ulcers persist at an incidence of $50 \%$.

After establishing the acetic acid ulcer model, I (SO) discovered a manuscript written by Robert \& Selye ${ }^{7)}$ describing a method that induced penetrating gastric ulcers via injection of a formaldehyde solution into rat stomach wall. We confirmed that such a method produced mucosal necrosis in the area of the injection. Even $6 \mathrm{~d}$ after injection, however, the necrotic tissue was still observed at the mucosal surface, such that production of a gastric ulcer was not achieved. In contrast to acetic acid ulcers, necrosis remained confined to the gastric mucosa without producing an ulcer crater.

In general, animals tolerated the acetic acid ulcer procedure well, without unfavorable effects. Occasionally, animals subjected to the injection of the acetic acid solution died due to gastric perforation on the second or third day after opera-

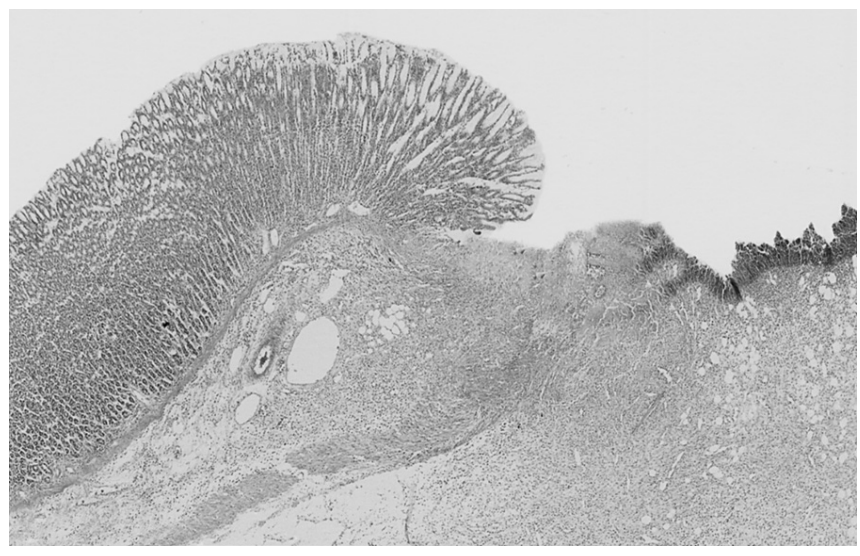

Fig. 4. Histological Appearance of Rat Stomach Acetic Acid Ulcer (day 5)

tion. All rats that lived for greater than $5 \mathrm{~d}$ after operation survived for the remainder of the experiment. Following this procedure, the external surface of the ulcerated region strongly adhered to the liver. Such adhesion appeared to represent the only shortcoming of this acetic acid ulcer model, as, in humans, the ulcer base seldom adheres to surrounding organs.

2.2. Type 2 Ulcer Model In 1969, I (SO) worked under the supervision of Professor James L. A. Roth at the University of Pennsylvania (Institute of Gastrointestinal Disease, Presbyterian Hospital) as a post-doctoral fellow. Since duodenal ulcers more frequently develop than gastric ulcers in the U.S.A., Dr. Roth asked me to establish an experimental duodenal ulcer model in rats. Although I diligently attempted to develop duodenal ulcers by injecting acetic acid solution into the duodenal wall, producing duodenal ulcers by an injection procedure proved quite difficult. Due to the precision 
Intraluminal application (3)

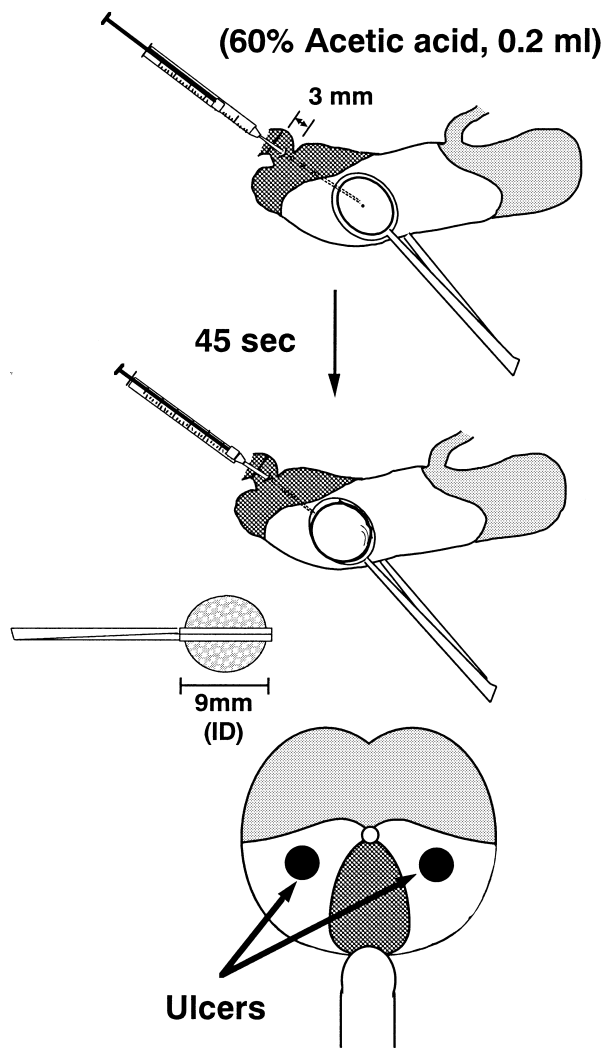

Intraluminal application (4)

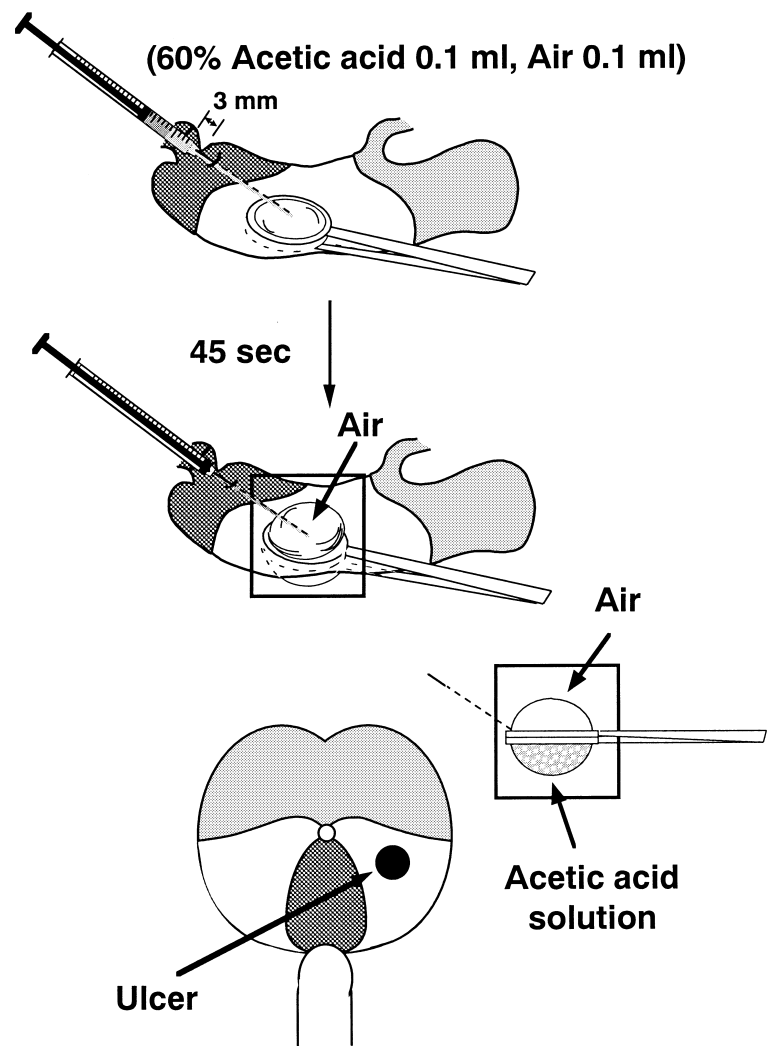

Fig. 5. Schematic Drawing of Experimental Procedure to Produce Acetic Acid Ulcers in Small Animals

(3) Intraluminal application of acetic acid solution into the rat stomach (Type 3 ulcer). (4) Intraluminal application of acetic acid solution and air (Type 4 ulcer).

required to inject acetic acid solution into the relatively thin duodenal mucosa, this procedure was fraught with difficulties. During one trial, we happened to observe blanched duodenal serosa after spilling acetic acid solution at the time of a failed acetic acid injection into the duodenal wall. When we examined the duodenal mucosa, there was hemorrhagic damage in the area corresponding to the blanched serosa. Considering such changes in the serosa and mucosa, Dr. Carl J. Pfeiffer (Head of the Institute) and I eventually devised a new method to induce duodenal ulcers using limited, focal application $(0.2 \mathrm{ml})$ of $100 \%$ acetic acid to the serosal surface for 30 to $60 \mathrm{~s}$ (Fig. 1). Clearly, this method is not limited to the duodenum, but can also be used for gastric ulcer induction (Fig. 5). This procedure subsequently became known as the Type 2 acetic acid ulcer model. Due to the relative simplicity, the Type 2 ulcer model is now used throughout the world more than the submucosal injection method ${ }^{8)}$ (Fig. 6). The Type 2 ulcer model has the similar shortcoming as the Type 1 ulcer model, i.e. the ulcer base adheres to the surrounding organs, notably the liver. Tarnawski et al. ${ }^{9)}$ demonstrated that vascular and microvascular changes that precede glandular cell necrosis represent a principal step in development of Type 2 acetic acid ulcers in rats. Bui et al. ${ }^{10)}$ also examined the histologic and ultrastructural features of the development, evolution, and healing of Type 2 duodenal ulcers in rats. Bui concluded that this reproducible and standardized model of duodenal ulcers begins with vascular injury as the earliest microscopic event. Subsequently, ischemic necrosis leads to

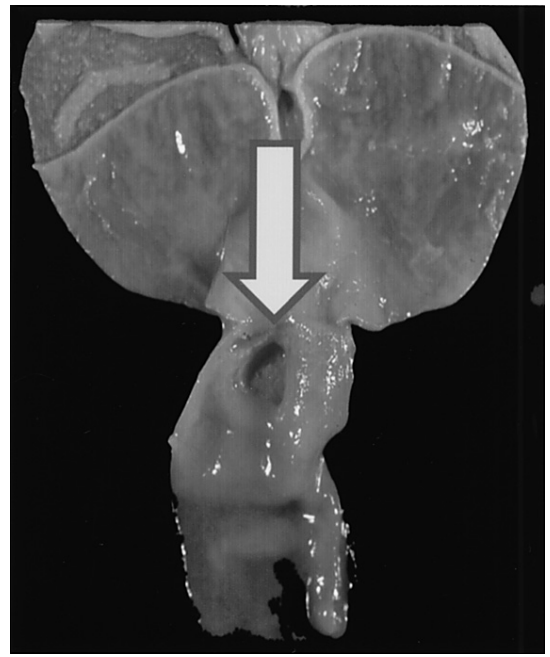

Day 7

Fig. 6. Gross Appearance of Rat Duodenal Ulcer Induced by Serosal Application of Acetic Acid Solution (day 7)

ulceration. Lastly, it deserves note that the chronic phase morphologically resembles human duodenal ulcers. With the experimental procedures for Type 1 and Type 2 ulcer models, ulcers were produced in mice, Mongolian gerbils (M. gerbils), ${ }^{11)}$ ferrets, guinea pigs, cats, ${ }^{12-14)}$ dogs (Figs. 7, 8), ${ }^{15-17)}$ and miniature pigs. ${ }^{18)}$ In dogs and pigs, gastric ulcers were 

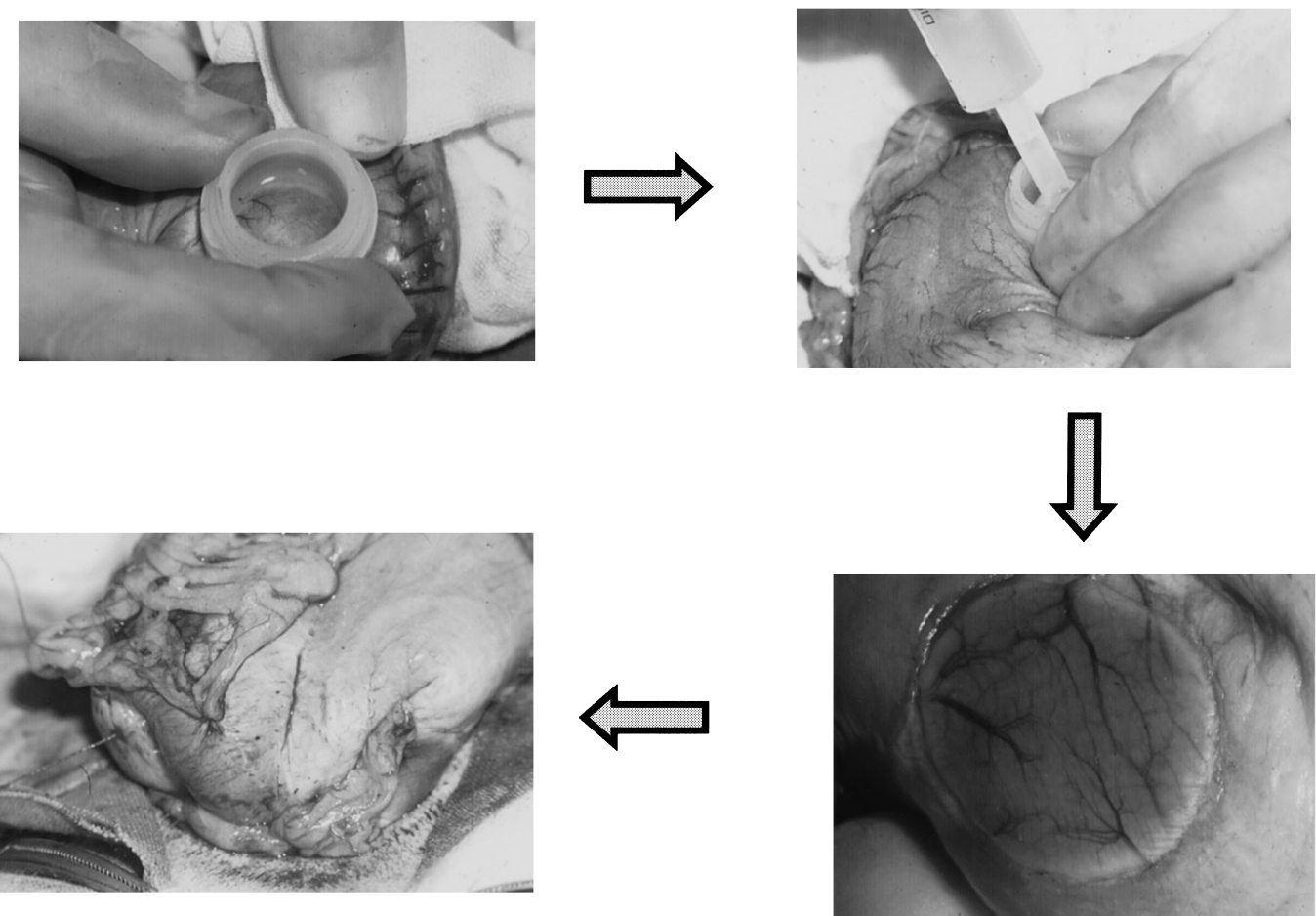

Fig. 7. Experimental Procedure to Produce Gastric Ulcer in Dog Stomach by Serosal Application of Acetic Acid Solution Heavy thrombosis is seen through the serosal site of the stomach after acid application.
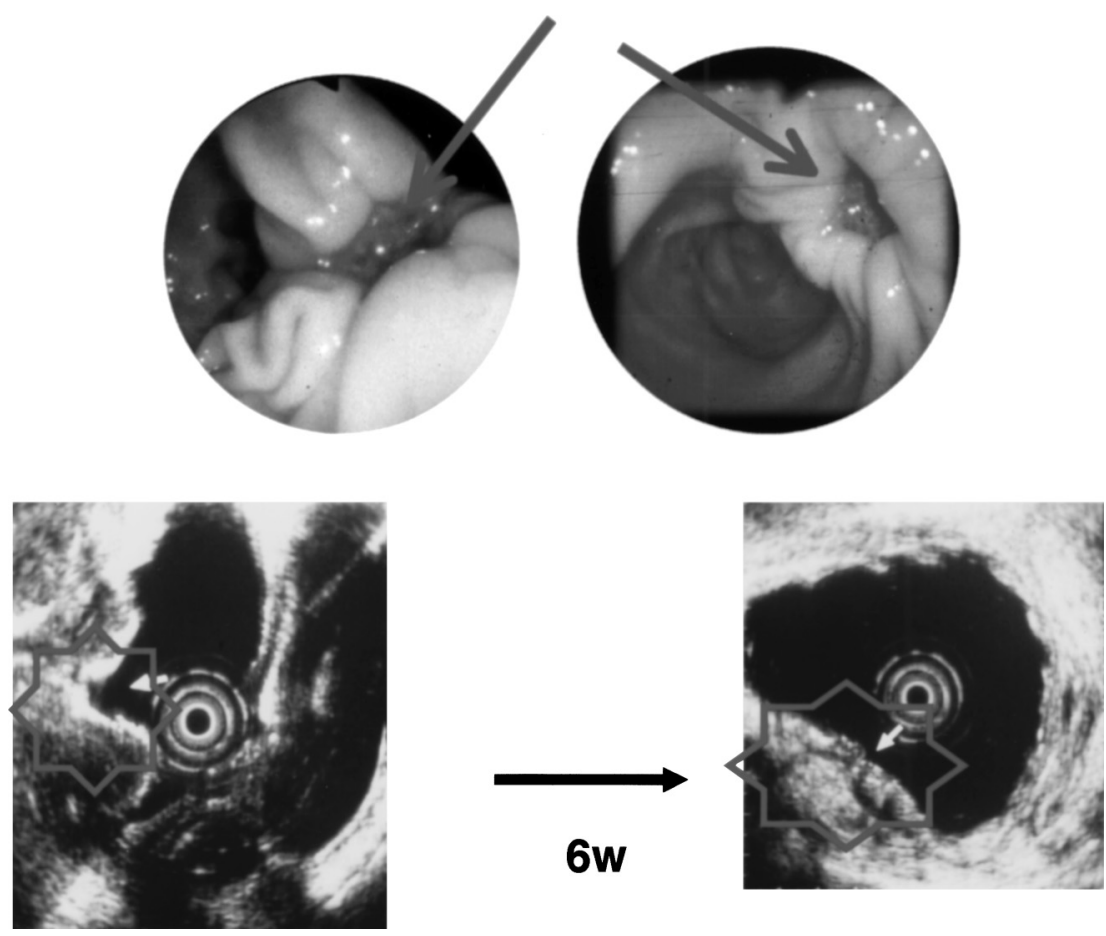

Fig. 8. Gross Appearance of Dog Stomach Acetic Acid Ulcers That Were Induced by Endoscopic Injection of Acetic Acid Solution (day 5) Endoscopic ultrasound appearance of such gastric ulcers.

easily produced by an endoscope equipped with an injection needle to inject $40 \%$ acetic acid $(1 \mathrm{ml})$ into the desired stomach wall region. Accordingly, ulcers could be produced without performing a laparotomy. Moreover, the gastric ulcers could be examined by direct endoscopic visualization or endoscopic ultrasound at appropriate intervals. Acetic acid ulcers in dogs completely healed within 6-7 weeks and did not relapse when observed for 13 weeks. Even in pigs, healing of acetic acid ulcers occurred within $30 \mathrm{~d}$. Similar to dogs and pigs, ulcers induced in other animals spontaneously healed and did not relapse, which is in distinct contrast to ulcers induced in rats.

2.3. Type 3 Ulcer Model The adhesion problem in Type 1 and Type 2 ulcer models necessitated exploration of 
alternative methods. For more than 25 years, the problem of adhesion proved an encumbrance. I attempted many procedures to eliminate such adhesion between the ulcer base and the liver, but was unable to meet with success. One attempted method was backing up the ulcer base with a portion of forestomach excised from the stomach of the same animal. As expected, adhesion between the ulcer base and the liver was prevented by insertion of fore-stomach tissue. Nonetheless, the procedure required additional time for routine ulcer induction. It was of interest, however, that the ulcer base, which usually consists of granulation tissue or newly formed connective tissue, could be replaced by the attached forestomach. With this chance observation, the adhesion problem neared resolution. It is of note that the healing of such ulcers was prolonged compared to ulcers that adhered to liver, probably due to decreased contraction of the ulcer base resulting from the fore-stomach tissue.

Subsequent research that the doctoral candidate Yasuhiro Tsukimi and I performed with chemical antrectomy provided a much needed clue for establishing a method devoid of the drawback of adhesion. The gastric antrum plays an important role in maintaining the integrity of the gastrointestinal tract via endogenous secretion of the trophic hormone gastrin. To elucidate the role played by gastrin in the healing of gastric ulcers, we removed the entire antral mucosa by chemical ablation. The antrum was first clamped using square-shaped forceps and acetic acid solution was then injected into the clamped area through the fundic mucosa for approximately $30 \mathrm{~s}$. Three days later, most of the antral mucosa was severely damaged and the serum gastrin level was greatly reduced. This finding allowed us to establish a new gastric ulcer model by means of intraluminal application of acetic acid solution. For that purpose, round forceps (ID $9 \mathrm{~mm}$ ) were developed to clamp the fundic area. Acetic acid solution was injected into the clamped portion through the distal antrum (Fig. 5). As expected, two deep, round ulcers, one on the anterior wall and the other on the posterior wall, developed in the area that had been exposed to the acetic acid solution. Due to the position of the ulcers that developed, we named these ulcers "kissing ulcers" (Fig. 9). ${ }^{19-21)}$ Such ulcers are occasionally observed in humans. To our surprise, the bases of such ulcers do not adhere to the liver or any other surrounding organ. This ulcer model is therefore more similar to human ulcers than Type 1 or Type 2 ulcer models. Nonetheless, in contrast to Type 1 and Type 2 ulcer models, the Type 3 ulcer model completely healed within 6 to 8 weeks after acid application and failed to exhibit relapse. The reason that Type 3 ulcers completely heal remains unclear, but we postulate that the lack of adhesion at the ulcer base results in permanent healing. Discovery of an ulcer model that closely resembles human ulcers by exhibiting both relapse and lack of adhesion at the ulcer base clearly represents the next model that needs to be developed. Nevertheless, this ulcer model can be particularly useful for measuring mucosal blood flow by the hydrogen gas clearance method. Due to its lack of adhesion, the mounting of stomachs with Type 3 ulcers to Lucite chambers is easier than with Type 1 or Type 2 ulcers. Our group ${ }^{22)}$ also examined the changes in gastric function and healing of acetic acid gastric ulcers in older rats. We found that aging had little or no influence on the development and healing rate of gastric ulcers, but the quality of ulcer healing was quite

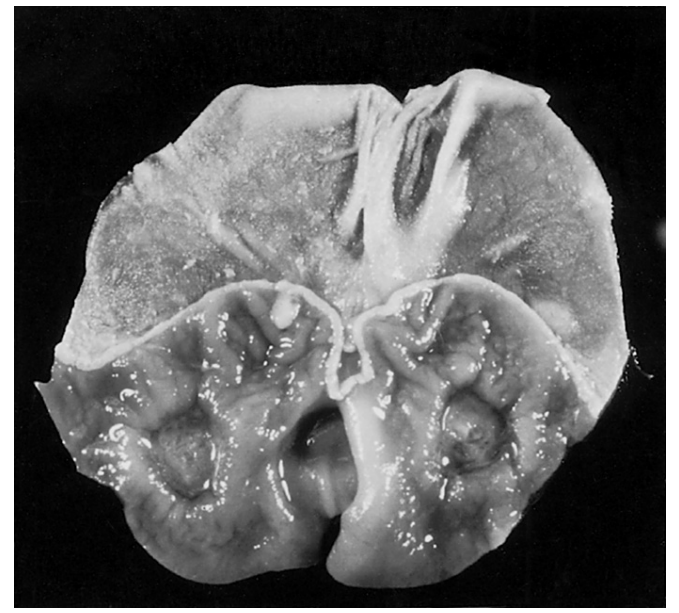

Fig. 9. Gross Appearance of Rat Stomach Acetic Acid Ulcers (Type 3, Kissing Ulcers), 3 d after Acid Application; Published Figure in Jpn. J. Pharmacol. 66, pp. 108 (1994).

different between younger ( 2 month old) and older (2426 month old) rats. Such a phenomenon might result from senescent decline in gastric function, such as decreased mucosal blood flow or mucosal cell proliferation in older rats.

Penney et al. $^{23)}$ examined whether or not age exerts an influence on development and natural or delayed healing of acetic acid ulcers in rats. Penney concluded that there were age-related differences in the development of gastric ulcers, but no age-related differences in natural and indomethacindelayed ulcer healing.

2.4. Type 4 Ulcer Model In order to produce a single ulcer via intraluminal application of acetic acid, we slightly modified our procedure for Type 3 ulcer model as follows. First, the anterior and posterior walls of the gastric corpus are clamped together with forceps that are used for the Type 3 ulcer model. A mixture of $60 \%$ acetic acid $(0.1 \mathrm{ml})$ and $0.2 \mathrm{ml}$ of air is then injected into the clamped lumen with an injection needle through the distal antrum, approximately 3 mm proximal to the pylorus. ${ }^{24)}$ The clamped stomach is horizontally positioned so that the injected air rises to the upper half and the acetic acid solution gravitates to the bottom half. Given such positioning, the acetic acid solution only contacts the lower half of the clamped mucosa, resulting in development of a single ulcer in the posterior mucosa. After $45 \mathrm{~s}$, the mixture of acetic acid solution and air is removed from the stomach and the abdomen is closed. A clearly defined deep, round ulcer consistently develops in the corpus of only the posterior wall $3 \mathrm{~d}$ after acid application; the anterior mucosa remains essentially intact in each animal.

\section{CHARACTERISTIC FEATURES OF ACETIC ACID ULCERS}

3.1. Spontaneous Relapse Although ulcers that develop in humans spontaneously heal with time, there is a high incidence of relapse after healing. The repeated pattern of healing and relapse turns a benign disease into a true nuisance. In contrast, most experimental ulcers induced by thermocautery, cryo-methods, or stomach clamping together with repeated cortisone treatment rapidly heal within 3 to 5 weeks after ulcer induction and fail to relapse. The lack of relapse 


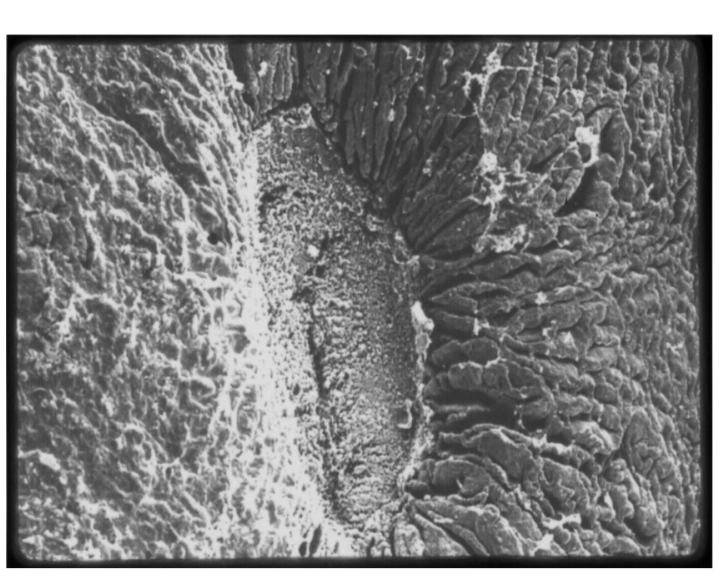

Day 60

\section{Starting Point of Relapse?}

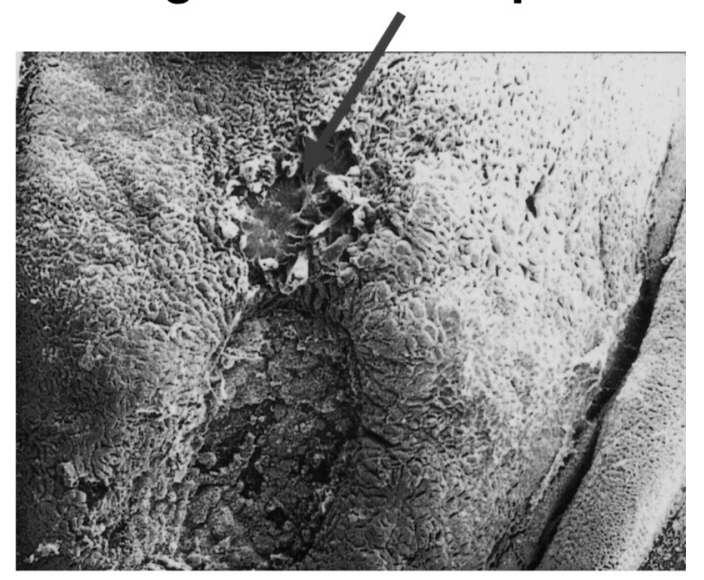

Day 100

Fig. 10. Scanning Electron Microscope Appearance of Rat Stomach Acetic Acid Ulcers (Type 1 Ulcers)

A portion of the edge of the gastric ulcer remained damaged $100 \mathrm{~d}$ after ulcer induction.

in such models prevented the study of the mechanisms underlying relapse with animal experiments for years. Similar to other ulcer models, acetic acid ulcers also rapidly diminish in size and depth within 2 weeks after ulceration. Nonetheless, we frequently noticed large ulcers that were bigger than the initial ulcers more than $100 \mathrm{~d}$ after ulcer induction. This finding strongly suggested that relapse of healed ulcers occurred, similar to the disease course observed in humans. With a scanning microscopic study, we found that the epithelial layer around the ulcerated area became damaged, which likely represents the initial step of spontaneous relapse (Fig. 10). Fukawa et al. ${ }^{25)}$ and Uchida et al. ${ }^{26)}$ reported that with endoscopic observation over 1.5 years, acetic acid ulcers induced in rats did indeed relapse after initially healing. Although time-consuming research will be requisite, the study of the mechanisms underlying ulcer relapse in rats represents an untapped research topic that will provide crucial data for elucidating ulcer pathophysiology. Arakawa et al. $^{27)}$ examined whether or not the administration of indomethacin beginning $4 \mathrm{~d}$ after acid injection and continuing for $56 \mathrm{~d}$ influences the healing and recurrence of acetic acid ulcers in rats. Following endoscopic examination of ulcers up to $240 \mathrm{~d}$, Arakawa found that the cumulative ulcer relapse rate was significantly higher in rats treated with indomethacin. In addition, ornoprostil, a PG analog, reversed the effects of indomethacin. Arakawa concluded that initial treatment with indomethacin promoted persistent polymorphonuclear cell infiltration and increased ulcer relapse, possibly via a PG-dependent mechanism.

3.2. Delayed Ulcer Healing Although the problem of chronicity, to include ulcer relapse, represents an important subject in the study of ulcers, experimental research on this subject remains limited.

3.2.1. Stress Physiologic or physical stress is well known to evoke gastric ulcers in both experimental animals and humans. In addition, stress might also contribute to gastric ulcer recurrence in humans. We studied the sensitivity of acetic acid ulcers induced in rats to stress in order to determine whether or not stress exerts an effect on the ulcer heal- ing process. ${ }^{28)}$ Water-immersion stress $(20 \mathrm{~h})$ performed at various intervals after acetic acid ulcer induction evoked stress ulcers in the glandular portion, but did not aggravate the acetic acid ulcers. Intermittent cold stress $\left(4^{\circ} \mathrm{C}\right) 10 \mathrm{~h}$ daily for 1 week also failed to exacerbate the acetic acid ulcers.

3.2.2. Anti-inflammatory Drugs Steroidal and nonsteroidal anti-inflammatory drugs (NSAIDs) frequently result in delayed ulcer healing, as well as damaged gastrointestinal mucosa.

Steroids Several groups ${ }^{29-31)}$ have reported that ulcers induced in rats by thermocauterization or stomach clamping with metal instruments are aggravated by cortisone treatment, becoming chronic. In addition, Kahn et al., ${ }^{32)}$ using cauterization ulcers in rats, reported that completely healed ulcers relapsed with cortisone treatment. Indeed, intramuscular administration of cortisone $(25 \mathrm{mg} / \mathrm{kg})$ for $5-20 \mathrm{~d}$ resulted in a high incidence of free perforation of acetic acid ulcers. In contrast, the same dose of cortisone given for 5$10 \mathrm{~d}$ beginning $50 \mathrm{~d}$ after operation did not result in aggravation of the gastric ulcers. Hase et al. ${ }^{33)}$ reported that prednisolone, administered at a dose of 20 or $40 \mathrm{mg} / \mathrm{kg}$ for $10 \mathrm{~d}$ after acid injection, significantly delayed the healing of acetic acid ulcers, probably by preventing angiogenesis in the granulation tissue at the ulcer base. Kuwayama et al. ${ }^{34)}$ found that delayed ulcer healing caused by hydrocortisone $\mathrm{Na}$ could be overcome by treatment with sucralfate, omeprazole, and TRY-200, a stable $\mathrm{PGI}_{2}$ analogue; nonetheless, the mechanism for such findings remains unknown.

We also found that prednisolone, when administered for 1 or 2 weeks after ulceration, significantly delayed ulcer healing and reduced the amount of collagen-containing tissue in the ulcer base. ${ }^{35)}$ Luo et al. ${ }^{36)}$ demonstrated that dexamethasone significantly delayed the healing of acetic acid ulcers in rats in association with inhibited angiogenesis at the ulcer margins and base. Ulcer induction significantly increased expression of basic fibroblast growth factor (bFGF), vascular endothelial growth factor (VEGF), and prostaglandin (PG) $\mathrm{E}_{2}$, as well as enhanced angiogenesis at the ulcer margins and 
base. After dexamethasone treatment, there was a significant decrease in $\mathrm{PGE}_{2}$ levels and VEGF expression, but not bFGF expression. Luo indicated that the delayed ulcer healing observed with dexamethasone might, at least in part, involve first reduced $\mathrm{PGE}_{2}$ levels, followed by down-regulation of VEGF.

NSAIDs Indomethacin, a potent non-steroidal anti-inflammatory drug, is known to delay the healing of experimental gastric ulcers in rats. ${ }^{37,38)}$ We also found that repeated administration of indomethacin at a dose of 1 to $3 \mathrm{mg} / \mathrm{kg}$ once daily or $1 \mathrm{mg} / \mathrm{kg}$ twice daily for 2 or 4 weeks consistently delayed the healing of acetic acid ulcers. ${ }^{39,40)}$ In contrast to cortisone treatment, free perforation of ulcers was not observed with indomethacin treatment for up to 8 weeks. It is also of note that Satoh's group ${ }^{41,42)}$ discovered that indomethacin treatment for 1 week beginning 6 weeks after acetic acid ulcer induction apparently induced a relapse of healed ulcers. Histologic studies suggest that the mechanism underlying delayed ulcer healing observed with steroids and indomethacin could be partially explained by inhibited ulcer base contraction due to decreased collagen-containing connective tissue. In contrast, prednisolone reduced connective tissue formation, such that the ulcer base could not contract to diminish its size. Accordingly, both indomethacin and prednisolone delay ulcer healing, but the mechanism of action for each drug appears to differ. After pre-treatment of rats with indomethacin for $5 \mathrm{~d}$, acetic acid injection into the submucosal area resulted in development of much larger ulcers compared with rats not receiving pre-treatment (unpublished data). This suggests that endogenous PG and related compounds greatly contribute to gastric mucosal maintenance and prevention of mucosal injury. Hirose et al. ${ }^{43)}$ examined the effects of indomethacin on fibroblast kinetics in ulcer healing in rats. In indomethacin-treated rats, fibroblasts, i.e., prolyl 4-hydroxylase and alpha-smooth muscle actinpositive spindle-shaped cells, minimally proliferated, with delayed appearance within ulcer tissue. Lyons et $a l .{ }^{44)}$ examined changes in sulfated macromolecules produced in vivo during normal and indomethacin-delayed healing of acetic acid ulcers in rats. Lyons found that indomethacin results in significant rises in sulfated glycosaminoglycan synthesis (chondroitin sulfate and dermatan sulfate) at the ulcer area compared with non-treated animals. Accordingly, Lyons suggested that increased glycosaminoglycan synthesis might be involved in the mechanism underlying delayed ulcer healing induced by indomethacin. The same group ${ }^{45}$ also demonstrated that, although indomethacin delays ulcer healing by impairing mucosal regeneration, it did not inhibit epithelial cell proliferation.

Naito et $a l .{ }^{46)}$ examined whether or not free radical scavengers exert any effect on indomethacin-induced delayed healing of acetic acid ulcers in rats. Rats were treated with scavengers for 6 weeks after ulcer induction; indomethacin treatment was subsequently performed. Superoxide dismutase did not affect the ulcer area after indomethacin treatment, yet allopurinol slightly inhibited increases in ulcer size. Dimethylsulfoxide produced a significant decrease in ulcer size, most probably by suppressing increases in lipid peroxides resulting from indomethacin. Naito concluded that lipid peroxidation mediated by oxygen radicals plays an important role in the mechanism of ulcer aggravation induced by indomethacin.

In addition to indomethacin, aspirin is also known to induce gastric mucosal damage and delay ulcer healing in humans and animals, ${ }^{47)}$ most likely by inhibiting cyclooxygenase $(\mathrm{COX})$ activity and reducing gastric PG levels. Brzozowski et al ${ }^{48)}$ examined the effects of a new class of aspirin, i.e., NO-releasing aspirin (NO-aspirin), on the healing of acetic acid ulcers in rats. Konturek found that while aspirin significantly delayed the healing of gastric ulcers, NO-aspirin did not affect ulcer healing. Konturek suggested that the NO released from NO-aspirin compensated for the PG deficiency induced by aspirin. Slomiany's group ${ }^{49)}$ reported that aspirin delays healing of buccal ulcers in rats by upregulating TNF $\alpha$, leading to amplification of apoptotic events and potentiation of the mucosal inflammatory responses that interfere with the healing process. Trefoil peptide is a mucin-associated regulatory peptide that is widely distributed in gastrointestinal tissue and acts to maintain mucosal integrity. Upon induction of acetic acid ulcers in rats, Ulaganathan et $a l .{ }^{50)}$ demonstrated that trefoil peptide and mRNA levels are significantly increased between 4 and $28 \mathrm{~d}$, with expression extending beyond the epithelium and primarily localizing to mucin-producing cells deep within the repairing mucosa. Such results strongly suggest that trefoil peptides are potential facilitators of late stage-repair processes.

3.2.3. Unhealed Gastric Ulcers Our group reported that 4-week treatment with indomethacin results in "unhealed ulcers" that persist for up to 12 weeks after cessation of treatment. ${ }^{35)}$ Such "unhealed ulcers" appear to be quite sensitive to mucosal protective drugs, such as sucralfate. Nonetheless, such ulcers are considerably resistant to anti-secretory drugs, such as omeprazole, despite the fact that gastric acid secretion in animals with "unhealed ulcers" was nearly completely inhibited. The reason why such "unhealed ulcers" could not heal despite cessation of indomethacin treatment remains unknown. We characterized such an "unhealed ulcer" model by assessing $\mathrm{PGE}_{2}$ biosynthesis, inflammation degree, and angiogenesis in the ulcer base. Ulcerated tissue $\mathrm{PGE}_{2}$ levels were significantly lower during indomethacin treatment, but the levels gradually returned to values comparable to those measured in the vehicle-treated control group. The results indicate that delayed healing is unrelated to endogenous PG levels in the stomach. Myeloperoxydase (MPO) activity levels were significantly higher during indomethacin treatment; such levels persisted after treatment cessation. Histologically, greater degrees of fibrosis and neutrophil accumulation, as well as a lesser degree of angiogenesis, were observed in the unhealed gastric ulcers compared to ulcers that healed in a normal fashion. It was concluded that severe fibrosis, persistent neutrophil infiltration, and poor angiogenesis in the ulcer base represent factors that are involved in the mechanisms underlying production of "unhealed gastric ulcers".

3.2.4. COX-2 and PAF Inhibitors and Platelets Sanchez-Fidalgo et al. ${ }^{51,52)}$ found that both ibuprofen (nonselective cycloxygenase inhibitor) and rofecoxib significantly delayed healing of acetic acid ulcers in rats. To evaluate the underlying mechanisms, they examined changes in two growth factors, as well as effects on angiogenesis around the ulcer by immunohistochemical methods. They found that both bFGF and VEGF levels were enhanced and microvasculature was increased in the granulation tissue of the ulcer 
bed. In addition, it was confirmed that proliferation and apoptosis were increased in the control groups. Rofecoxib was able to significantly reduce such proliferation and apoptosis. It is of interest that while rofecoxib reduced bFGF expression, VEGF expression was not affected. Moreover, development of new microvasculature was also not affected. Ibuprofen had no effect on any of the assessed factors. Furthermore, rofecoxib significantly inhibited increased cellular proliferation and apoptosis in the ulcer area. Accordingly, it was concluded that reduced bFGF expression, an antiangiogenic effect, and proliferation/apoptosis inhibition are all possible mechanisms involved in delayed ulcer healing induced by rofecoxib. Berenguer et al. ${ }^{53)}$ also reported that cerecoxib, a selective COX-2 inhibitor, significantly delayed the healing of acetic acid ulcers in rats. Berenguer suggested that down-regulation of COX-2 expression is involved in the mechanism underlying delayed healing. Slomiany's group ${ }^{54)}$ found that platelet-activating factor (PAF) inhibitor, BN52020, significantly delayed the healing of acetic acid ulcers in rats, possibly due to decreased COX-2 protein expression, an increased rate of epithelial cell apoptosis, and increases in TNF $\alpha$ and mucosal NOS-2 activity. Laudanno et $a l^{55)}$ reported that both celecoxib and rofecoxib significantly aggravated and complicated gastric ulcers induced by acetic acid methods.

Akiba et al. $^{56)}$ reported that both L-NAME and aminoguanidine significantly delay the healing of both gastric and duodenal ulcers, suggesting that NO plays a role in the healing of gastric ulcers. In contrast to the above findings, Araki et $a{ }^{57)}$ found that while both indomethacin and rofecoxib significantly delayed the healing of Type 2 gastric ulcers, the drugs did not delay healing of duodenal ulcers in rats. It is of interest that COX-2 mRNA expression is upregulated in the vicinity of gastric and duodenal ulcers. It is also of note that both drugs significantly suppressed the increased PG biosynthetic response, even in the vicinity of the duodenal ulcers. Baatar et al. ${ }^{58)}$ examined the effects of cerecoxib on the healing of esophageal ulcers induced by acetic acid in rats at the cellular and molecular level. Baatar found that cerecoxib significantly delayed the healing of esophageal ulcers by reducing esophageal epithelial cell proliferation, likely by downregulating the $\mathrm{HGF} / \mathrm{c}-\mathrm{Met}-\mathrm{Erk} 2$ signaling pathway. Shen ${ }^{59)}$ reported that NS-398 significantly delayed the healing of acetic acid ulcers in rats, possibly by suppressing increases in PG levels in the ulcerated gastric mucosa. Brzozowski et $a{ }^{60)}$ discovered that both aspirin and indomethacin significantly delay ulcer healing due to suppression of endogenous PG, impairment of gastric mucosal blood flow at the ulcer site, and excessive cytokine expression (IL- $1 \beta$, TNF $\alpha$ ) and release. Similar results were obtained with highly selective COX-1 (resveratrol) and COX-2 inhibitors (NS-398), suggesting that both $\mathrm{COX}$ isomers are important sources of $\mathrm{PG}$ that contribute to ulcer healing.

Ma et $a{ }^{61)}$ hypothesized that the noxious effect of aspirin on ulcer healing might be attributed to its anti-aggregatory effect on platelets. Ma suggested that anti-platelet drugs might interfere with gastric ulcer healing by suppressing release of growth factors, such as VEGF, from platelets. Concomitant with acetic acid ulcer induction, a significant increase in serum VEGF levels and a significant decrease in serum endostatin (an anti-angiogenic factor) levels were ob- served in rats. Ticlopedine (an ADP-receptor antagonist), but not aspirin, significantly impaired ulcer healing and angiogenesis, reversing such changes in serum VEGF and endostatin levels. Wallace concluded that drugs such as ticlopidine may exert their effects by altering the ability of platelets to release growth factors.

3.2.5. Diabetic Rats Konturek's group ${ }^{48,62)}$ recently reported that healing of Type 2 acetic acid ulcers induced in diabetic rats (resulting from streptozocin) was significantly prolonged compared with non-diabetic rats. Rats with delayed ulcer healing showed a reduction of gastric mucosal blood flow at ulcer margins. In addition, Konturek found a significant increase in pro-inflammatory cytokine (IL-1 $\beta$, TNF- $\alpha$, and IL-10) plasma levels, suppression of VFGF and platelet endothelial cell adhesion molecule-1 (PECAM-1), and mucosal over-expression of heat shock protein (HSP) 70. Both treatment with aspirin and rofecoxib significantly delayed ulcer healing in diabetic rats in association with extensively reduced gastric blood flow and increased plasma cytokine levels. Such data strongly suggest that the mechanism of delayed ulcer healing in diabetic rats involves deficient PG biosynthesis around the ulcer area. It is of note that NO-aspirin counteracted the aggravating effects of aspirin in diabetic rats. It is likely that released NO compensated for PG deficiency, resulting in enhancement in gastric blood flow at ulcer margins and suppression of cytokine release in the ulcer area. Delayed ulcer healing was reversed by administration of insulin and pentoxyfylline, an inhibitor of TNF $\alpha$.

3.2.6. Smoking A positive relationship between cigarette smoking and gastroduodenal ulcers has been empirically known for many years. Indeed, patients with ulcers are recommended to refrain from smoking. Nonetheless, the mechanism by which smoking results in noxious effects on ulcer healing remains unclear. Iwata and Leung ${ }^{63)}$ examined the effects of tobacco cigarette smoke on the healing of acetic acid ulcers in rats. They demonstrated that cigarette smoke, i.e. mainly nicotine, significantly attenuated ulcer margin hyperemia and resulted in increased ulcer size in the acute and healing stages. Such data suggest that the inhibition of hyperemia at ulcer margins is the mechanism underlying the aggravating effects of cigarette smoke. They evaluated the possible participation of endogenous PG metabolism in the hyperemia and increases in ulcer size. Such changes were significantly inhibited by treatment with exogenous PG (misoprostol). Subsequently, they concluded that the adverse effects of cigarette smoke on chronic ulcers might be mediated by inhibition of endogenous PGs.

Shin et $a l .{ }^{64)}$ elucidated the mechanism underlying the noxious effect of cigarette smoking on ulcer healing using acetic acid ulcers in rats. With administration of cigarette smoke extract (chloroform and ethanol), acetic acid ulcers became larger, which was followed by an increase in MPO activity and a reduction in cell proliferation. Such a smoke extract resulted in increased microvasculature around the ulcer area, following increases in bFGF. With an in vitro study, Shin demonstrated that smoke extract delayed cell migration and decreased cell proliferation in association with a reduction in ornithine decarboxylase activity. Such changes were reversed by exogenous spermidine. Shin concluded that delayed ulcer healing induced by cigarette smoking may, at least in part, be related to reduced polyamine synthase activity. 


\section{INFLUENCE OF H. pylori INFECTION}

4.1. Infection of Mice and Rats The discovery of Helicobacter pylori (H. pylori) by Warren and Marshall in $1983^{65)}$ and its recognition as the most important pathogenic factor involved in gastritis and peptic ulcers heightened our interest in ulcer research. To confirm the noxious effects of H. pylori on the stomach, various groups, including our own, introduced CagA and VacA positive H. pylori into mice, rats, M. gerbils, monkeys, and miniature pigs with and without ulcers. Bui et al. ${ }^{66)}$ were the first to report that $H$. pylori, inoculated once daily for $7 \mathrm{~d}$, could delay the healing of acetic acid ulcers induced in rats. We also administered H. pylori to rats with acetic acid ulcers and found that the bacteria tended to delay the healing of acetic acid ulcers for up to 10 weeks after inoculation. ${ }^{67)}$ In that study, MPO activity tended to be increased with $H$. pylori infection. The fact that antibiotics and acid pump inhibitors prevented delayed healing resulting from $H$. pylori have offered valuable insights into the mechanisms of ulcer healing. Konturek's group ${ }^{68,69)}$ reported that Cag A and Vac A positive H. pylori water extract could delay ulcer healing in mice and rats. They contend that the underlying mechanism involves reduction of gastric mucosal blood flow, over-expression of cytokines, such as IL- $1 \beta$ and TNF $\alpha$, and disruption of the balance between serum gastrin/somatostatin. In addition, Konturek's group found that both aspirin treatment and $H$. pylori infection significantly delayed the healing of acetic acid ulcers in rats. Nonetheless, $H$. pylori infection antagonized aspirin-induced delay of ulcer healing due to suppression of acid secretion, enhancement in $\mathrm{PGE}_{2}$ levels, and over-expression of TGF $\alpha$ and VEGF in the ulcer area. Gunawan et al. ${ }^{70)}$ also demonstrated that $H$. pylori infection significantly delayed the healing of acetic acid ulcers in mice, inhibiting angiogenesis at the ulcer margins. Our group found that colonization of $H$. pylori in mice and rats was possible, yet visible pathologic changes did not occur, even 6 months after infection (unpublished data).

The manifestation of gastritis or ulcer disease in $\mathrm{H}$. pylori infected individuals is less common when NSAIDs are administered. Accordingly, our group ${ }^{11)}$ examined the effects of indomethacin treatment on the inflammatory response caused by $H$. pylori in rats with acetic acid ulcers. Both $H$. pylori (ATCC43504, $\left.10^{8} \mathrm{CFU}\right)$ and indomethacin $(1$ or $2 \mathrm{mg} / \mathrm{kg}$ for 4 weeks) were orally or subcutaneously administered after ulceration. Indomethacin significantly delayed ulcer healing and increased MPO activity to the same degree in both $H$. pylori infected animals and non-infected animals. H. pylori infection exerted an insignificant effect on ulcer healing, but tended to increase MPO activity up to 4 weeks. Such results demonstrate that indomethacin treatment delayed ulcer healing, regardless of the presence or absence of $H$. pylori. Moreover, indomethacin treatment reduced the amount of $H$. pylori bacteria in the stomachs.

Hata et $a l .{ }^{71)}$ examined the effects of continuously administered $0.02 \%$ ammonia on the healing of acetic acid ulcers in rats. Ammonia treatment resulted in a significant increase in the ulcer index 4 and 8 weeks after ulcer induction. In addition, suppression of cell cycling of the regenerative epithelium and fibroblasts in the ulcer margin was observed, suggesting direct toxicity from ammonia. Kodama et $\mathrm{al}^{72)}$ induced acetic acid ulcers in the stomachs of Japanese mon- keys by endoscopic injection of acetic acid solution. The monkeys were subsequently infected with $H$. pylori. Although the ulcers in the monkeys spontaneously healed within 8 weeks, H. pylori infection clearly delayed ulcer healing. At that time, the ammonia concentration of the gastric secretions and the grade of inflammation were significantly increased in the $H$. pylori infected group compared with the non-infected control groups. It is well known that ammonia production is increased by the action of bacterial urease on urea in the gastric lumen.

4.2. Infection of $\mathbf{M}$. gerbils Hirayama et $a l^{73)}$ and other groups ${ }^{74,75)}$ to include our own, found that $H$. pylori can induce gastritis and gastric ulcers in M. gerbils with an incidence of $>80 \%$. Consequently, various groups have examined whether or not $H$. pylori exerts an influence on ulcer healing in gerbils. We examined how $H$. pylori infection, indomethacin, and their combination affects the healing of gastric ulcers and whether or not such factors provoke a relapse of healed gastric ulcers in M. gerbils. It was found that $H$. $p y$ lori infection significantly delayed ulcer healing in M. gerbils 4 weeks following infection. In addition, $H$. pylori infection resulted in a relapse of healed ulcers 1 to 6 months after inoculation, as well as a gradual increase in ulcer size. ${ }^{11)}$ Serum gastrin levels were significantly increased upon ulcer relapse. H. pylori-induced ulcers in the posterior wall developed in addition to relapsed ulcers 5 and 6 months after infection. Such studies clearly demonstrate that the M. gerbil model is useful for elucidation of the pathophysiology of active, healed, and relapsed ulcers involving $H$. pylori infection.

\section{DRUG TREATMENTS AND ULCER HEALING}

Needless to say, the development of pharmaceutical agents remains very difficult. Usually, drug development relies heavily on serendipity or logical alteration of existing drugs. In 1972, Black et $a{ }^{76}{ }^{76}$ logically developed several compounds that blocked histamine $\mathrm{H}_{2}$ receptors, resulting in marked inhibition of gastric acid secretion, both basal secretion and secretion stimulated by histamine, gastrin, carbachol, and food. Due to their powerful anti-secretory effects, such compounds significantly enhanced the healing of acidrelated diseases. With the development of such drugs, the rate of surgical intervention in ulcer disease was greatly reduced. Currently, $\mathrm{H}_{2}$-receptor antagonists are the most popular prescription for ulcer patients worldwide. The mechanisms underlying acid secretion have not been well documented. In particular, the final steps of hydrogen secretion have long remained an enigma. Ganser and Forte ${ }^{77)}$ found that there is an ATPase activated by $\mathrm{K}^{+}$ions in the stomach. Subsequently, it was demonstrated that the ATPase is $\mathrm{H}^{+}, \mathrm{K}^{+}$ATPase, which drives hydrogen ions from parietal cells into the gastric lumen. In Sweden, while researchers working at Astra Drug Co. were looking for locally active anti-secretory drugs, they found that benzimidazole derivatives potently inhibit acid secretion stimulated by histamine, gastrin, and carbachol. Even dibutylyl cAMP-stimulated secretion was inhibited by their compounds. ${ }^{78)}$ After careful examination, Astra workers, with the collaboration of Sachs, found that these compounds could inhibit gastric proton pumps. Four of these pump inhibitors, omeprazole, lansoprazole, rabeprazole, and pantoprazole are now available throughout the 
world as potent anti-ulcer drugs due to their prolonged antisecretory activity (over $24 \mathrm{~h}$ ). In dogs, we confirmed that the anti-secretory activity persisted for more than $30 \mathrm{~h}$.

\subsection{Anti-secretory Drugs}

H2-Blockers Since the establishment of acetic acid ulcer models, such models have found application in the screening of a number of compounds for development of ulcer-healing drugs. Upon discovery of $\mathrm{H}_{2}$-blockers, we examined their effects on the healing of acetic acid ulcers. Such drugs strongly inhibited gastric acid secretion and prevented the occurrence of acute gastric or duodenal ulcers induced by water immersion stress and NSAIDs. Nonetheless, most of the $\mathrm{H}_{2}$-blockers did not exhibit a definite effect on the healing of acetic acid ulcers, even upon administration of extremely high, frequent ( 4 times/d) doses. One possible explanation for this result is the lack of consistent inhibition of gastric acid secretion, as the drug displays only a limited duration of effectiveness that persists for 3 to $4 \mathrm{~h}$ following injection. Another consideration is that histamine has an ability to inhibit cell growth and induce apoptosis in tumor cell lines, ${ }^{79)}$ independent of $\mathrm{H}_{2}$-receptors. Accordingly, the relatively weak effect of $\mathrm{H}_{2}$-blockers on ulcer healing might be related to such pharmacologic effects that do not favor ulcer healing. Tsuchida et al. $^{80)}$ examined the effects of cimetidine and omeprazole on the healing of acetic acid ulcers in rats and angiogenesis. It was found that oral cimetidine may inhibit angiogenesis in ulcer granulation tissue by blocking histamine $\mathrm{H}_{2}$ receptors, resulting in an unfavorable effect on ulcer healing.

Proton Pump Inhibitors The ulcer healing effects of proton pump inhibitors, such as omeprazole, lansoprazole, pantoprazole, were confirmed in experimental animals by various research groups, including our own. ${ }^{53,81-83)}$ It should be noted that Takeuchi et al. ${ }^{84)}$ reported that portal hypertension significantly inhibited epithelial cell proliferation at the ulcer margins, delaying the healing of acetic acid ulcer in rats. Nonetheless, omeprazole treatment significantly accelerated ulcer healing in both normal rats and rats with portal hypertension to a similar extent. Since serum gastrin levels increased with omeprazole treatment, Takeuchi postulated that epithelial cell proliferation and ulcer healing results from a trophic effect of increased serum gastrin. Ishihara and Ito ${ }^{85}$ found that while both cimetidine and omeprazole enhanced the healing of acetic acid ulcers in 8- and 48-week old rats, the drugs could not enhance ulcer healing in 96 week-old rats. They contend that 8 - and 48-week old rats exhibited significantly increased serum gastrin levels, while 98-week old rats did not. The failure to enhance ulcer healing was thought to result from the lack of an elevated gastrin level. They postulate that the positive effect on ulcer healing of the two drugs is strongly related to gastrin blood levels.

We demonstrated that omeprazole significantly enhanced spontaneous healing of acetic acid ulcers and prevented the indomethacin-induced delay in ulcer healing. ${ }^{81,82)}$ Kuwayama et $a l .{ }^{86)}$ reported that lansoprazole also significantly prevented the delayed ulcer healing caused by hydrocortisone. Ramaswamy ${ }^{87)}$ reported that oxytocin significantly enhanced the healing of acetic acid ulcers in rats, possibly by inhibiting gastric acid secretion. Atosiban, an oxytocin receptor antagonist, reversed the ulcer healing effect of oxytocin, suggesting involvement of oxytocin receptors in ulcer healing.

\subsection{Mucosal Defensive Drugs}

Sucralfate Sucralfate (sucrose aluminum sulfate) is a common drug that has been shown to enhance ulcer healing without affecting gastric secretion. We repeatedly confirmed the ulcer healing activity of sucralfate in rats; the effect was similar to that of acid pump inhibitors. ${ }^{88,89}$ ) The effective dose of sucralfate on ulcer healing was far higher (600 to $1200 \mathrm{mg} / \mathrm{kg} / \mathrm{d})$ than that of omeprazole $(10-30 \mathrm{mg} / \mathrm{kg} / \mathrm{d})$, due to the fact that the effect is local. The underlying mechanism remains unclear, but it has been reported that sucralfate selectively binds to an ulcer base protein, creating a "bandaid" effect. Tsukamoto et al. ${ }^{90)}$ demonstrated that sucralfate significantly enhanced the healing of acetic acid ulcers and increased the extent of angiogenesis in the ulcer base $10 \mathrm{~d}$ after ulceration in rats. In contrast, cimetidine decreased the extent of angiogenesis. Simultaneous treatment of sucralfate and cimetidine enhanced ulcer healing, yet the extent of angiogenesis was not affected by the combination of drugs. Such data suggest that the effect of sucralfate is partly due to stimulation of angiogenesis. Szabo et al. ${ }^{91,92)}$ suggested that sucralfate might trap epidermal growth factor in the stomach, thereby contributing to ulcer healing.

PGs A number of studies have been performed to clarify the cytoprotective effect of PGs. We examined the effects of $\mathrm{PGE}_{2}$ and its analog on ulcer healing. As expected, PG analogs significantly enhanced the spontaneous healing of acetic acid ulcers and prevented a delay in ulcer healing by repeated indomethacin treatment. ${ }^{93-97)}$ Penney et al. ${ }^{98)}$ demonstrated that misoprostol reversed the delayed healing effect of aspirin via an effect on epithelial regeneration. Arakawa et al. ${ }^{99)}$ examined the effects of rebamipide, a novel PG-inducer, on healing and relapse of acetic acid ulcers in rats. Arakawa found that rebamipide and rebamipide together with cimetidine resulted in a higher healing rate and a lower cumulative relapse rate compared to control groups. Arakawa suggested that rebamipide enhances ulcer healing and decreases ulcer relapse.

Growth Factors. Epidermal Growth Factor (EGF) The effects of EGF on the healing of acetic acid ulcers have been confirmed by various groups. ${ }^{100-103)}$ We found that orally administered hEGF for 2 weeks significantly enhanced acetic acid ulcer healing in rats. ${ }^{104)}$ In addition, it was found that b-FGF also significantly enhanced ulcer healing (unpublished data). Satoh et $a l_{.}{ }^{42}$ ) have also elucidated that bFGF markedly enhanced acetic acid ulcer healing in rats. The major source of EGF is submandibular glands, such that submandibular gland resection might delay ulcer healing due to a lack of EGF. Nonetheless, we found that submandibular resection had little to no effect on ulcer healing for up to 8 weeks following operation (unpublished data). Accordingly, it remains unclear whether or not EGF secreted from the submandibular glands plays a role in ulcer healing. Chao's group $^{105)}$ reported that oral EGF significantly accelerated duodenal ulcer healing in rats. Perez-Aisa et al. ${ }^{106)}$ found that both exogenously administered PDGF and EGF significantly enhanced duodenal ulcer healing in rats and reversed the indomethacin-induced delay in ulcer healing. An increase in collagen proliferation and secretion, which does not affect gastric acid secretion, was postulated as the underlying mechanism. The same study demonstrated that famotidine also accelerated duodenal ulcer healing and reversed the ef- 
fects of indomethacin by achieving marked inhibition of gastric acid secretion and collagen secretion by granulation tissue. Mine et $a l .{ }^{107)}$ found that both cimetidine and EGF significantly enhanced the healing of acetic acid ulcers in rats and increased connexin 32 expression at ulcer margins. Mine suggested ulcer healing was linked to connexin formation. Wingren et al. ${ }^{108)}$ examined the effects of excision of the submandibular salivary glands and the role of gender on healing of acetic acid ulcers in rats. Excision of the glands delayed ulcer healing in both male and female rats. Nonetheless, ulcer healing in female rats was slower than that of male rats. Konturek et al. ${ }^{109)}$ reported that sucralfate binds EGF in a $\mathrm{pH}$-dependent manner, leading to its accumulation in ulcerated areas. Accordingly, the peptide is locally available in high concentrations to accelerate tissue repair and the healing of ulcerated mucosa. Indeed, the ulcer healing effects of sucralfate were reduced with sialoadnectomy and partially restored with oral administration of EGF. Tarnawski et al. ${ }^{110)}$ immunohistochemically demonstrated increased expression of EGF receptors in the mucosal margins of acetic acid ulcers in rats.

Fujisawa et al. ${ }^{111)}$ reported that healing of acetic acid ulcers induced in rabbit oral mucosa was delayed after removal of submandibular glands or subcutaneous injection of cisplatin and peplomycin sulfate. Such delayed healing by gland removal or drug administration was reversed with either systemic treatment with bFGF and EGF or topical application of bFGF. Fujisawa concluded that bFGF might be effective for refractory oral mucosal ulcers.

bFGF Yabu et al. ${ }^{112)}$ confirmed the expression of bFGF in fibroblasts in ulcer beds $5 \mathrm{~d}$ after ulceration. It is postulated that similar to EGF, bFGF, as well as aFGF, enhance ulcer healing by stimulating fibroblast proliferation. ${ }^{113,114)}$ After 3 weeks, the ulcerated area surface was completely covered by regenerated epithelium. Gastric tissue was immunohistochemically negative for bFGF both inside and outside the scar region. Konturek et al. ${ }^{115)}$ examined the effects of bFGF and sucralfate on the healing of acetic acid ulcers in rats. Konturek found that both agents significantly enhanced ulcer healing in a dose-related manner. bFGF subcutaneously or intragastrically accumulated twice to three times as much in the ulcer area than in the intact mucosa, particularly in rats treated with sucralfate. Concurrent treatment with indomethacin delayed ulcer healing and reduced both the binding of labeled bFGF to the ulcer area and angiogenesis caused by sucralfate. bFGF appears to play an important role in the mechanisms of ulcer healing related to angiogenic activity.

Akiba et al. $^{116)}$ examined the effects of recombinant human bFGF (CS23) and Ng nitro-K-arginine methyl ester (L-NAME), an NO synthase (NOS) inhibitor, on the healing of acetic acid ulcers in rats. Akiba found that orally administered CS23 significantly enhanced ulcer healing, yet LNAME significantly delayed ulcer healing and inhibited the ulcer healing effect of CS23. In rats with ulcers, constitutive NOS (cNOS) activity significantly decreased and vessel and neuron cNOS immunoreactivity disappeared. Nonetheless, inducible NOS activity increased. In addition, they revealed that CS23 recovered NO synthesis in endothelial cells and neurons. Based upon such data, they suggested that increased NO synthesis with angiogenesis and re-innervation has a beneficial effect on gastric ulcer healing. Nakamura et al. ${ }^{117)}$ reported that during the natural healing process of acetic acid ulcers in rats, few autonomic nerves were recognized surrounding the newly formed arterioles and venules. When rats with ulcers were treated with CS23, the cholinergic, calcitonin gene-related peptide, and vasoactive intestinal peptideimmunoreactive nerves were clearly recognized near the microvasculature, but few adrenergic nerves were seen. Nakamura suggested that bFGF promotes re-innervation of the newly formed microvasculature.

Brzozowski et al. ${ }^{118)}$ clearly demonstrated that local injection of bFGF into acetic acid ulcer bases significantly enhanced ulcer healing, which was accompanied by an extensive rise in the gastric blood flow at the ulcer margins and a marked increase in the amount of microvasculature. In contrast, depletion of endogenous bFGF at the ulcer area by specific neutralizing antibodies delayed ulcer healing, reducing angiogenesis at the ulcer bed. Such results strongly indicate that bFGF is a necessary factor for ulcer healing due to its ability to induce angiogenesis. In addition, Brzozowski suggested that COX-2 derived PGs and suppression of gastric acid secretion may also play an important role in enhanced ulcer healing induced by various growth factors.

Satoh's group ${ }^{41,42)}$ reported a series of experiments on the relapse of healed acetic acid ulcers in rats by indomethacin treatment and prevention of such ulcer relapse by bFGF mutein (CS23). First, Satoh demonstrated that CS23 significantly enhanced ulcer healing by administering it for 4 weeks after ulceration. Second, indomethacin treatment for 2 weeks beginning 4 weeks after ulceration aggravated the nearly healed ulcers. Concurrent treatment with indomethacin and CS23 for 2 weeks beginning 4 weeks after ulceration significantly prevented ulcer relapse by indomethacin. Ranitidine, a histamine $\mathrm{H}_{2}$ blocker, also significantly prevented indomethacin-induced ulcer relapse.

Transforming Growth Factor $\boldsymbol{\beta}_{\mathbf{1}}$ (TGF $\left.\boldsymbol{\beta}_{\mathbf{1}}\right)$ TGF- $\boldsymbol{\beta}_{1}$ has been shown to play a central role in wound healing. As TGF$\beta_{1}$ is detected in the stomach, it is postulated that this cytokine is involved in the mechanisms underlying ulcer healing. As described above, indomethacin can delay the healing of acetic acid ulcers in rats. Perez-Aisa et al. ${ }^{101)}$ demonstrated that indomethacin significantly delayed the healing of duodenal ulcers induced by serosal application of acetic acid solution. It was noted that TGF $\beta$ and famotidine improved ulcer healing and reversed the adverse effects of indomethacin, possibly by causing increased epithelial and granulation tissue cell proliferation and decreased gastric acid secretion, respectively. Ernst et al. ${ }^{113,114)}$ reported that subserosal application of TGF- $\beta_{1}$ significantly enhanced acetic acid ulcer healing in rats, possibly by increasing both formation of granulation tissue and cell migration. Nonetheless, the healing effects of TGF- $\beta_{1}$ do not depend on a vascular factor. Ernst et al. ${ }^{115)}$ demonstrated that although subserosal injection of TGF- $\beta_{1}$ alone induced excessive scarring, concurrent treatment of TGF- $\beta_{1}$ neutralizing antibody also accelerated and improved acetic acid ulcer healing.

Hepatocyte Growth Factor (HGF) Upon sustaining an injury, the liver quickly recovers to its normal state through HGF secretion. The potential enhancement of ulcer healing by hepatectomy, in addition to enhanced healing of an injured liver through HGF, was examined in our laboratory 
(unpublished data). A hepatectomy (approximately 3/4 removal) or a laparotomy alone (control) was performed at the time of acetic acid ulcer induction. Upon sacrificing animals 1 and 2 weeks after ulceration, the ulcer size was determined and the HGF blood level was measured by ELISA. The liver recovered to a great extent within 1 and 2 weeks, i.e., the wet weight being $15 \mathrm{~g}$ and $20 \mathrm{~g}$, respectively, compared to $25 \mathrm{~g}$ in the controls. Nonetheless, gastric ulcer healing was not affected by partial hepatectomy, as assessed at 1 and 2 weeks. HGF levels were significantly increased during the first week, yet subsequently returned to control levels. Such results suggest that the mechanism of healing in the liver is completely different from that of gastric ulcer healing. Based upon detailed examination in rats, Udagawa et al. ${ }^{119)}$ suggested that the anti-ulcer effect of rebamipide involves upregulation of $\mathrm{HGF}$, c-met, $\mathrm{COX}-2$, and EP2 in the ulcer area. Brzozowski et al. ${ }^{120)}$ examined the effects of HGF and gastrin on acetic acid ulcer healing in rats by local injection of specific antibodies against HGF and gastrin just around the ulcer immediately after ulcer induction. Ulcer healing was significantly delayed due to the fall in the mucosal blood flow and decrease in COX-2 expression, leading to reduced $\mathrm{PG}$ levels at the ulcer margins. Brzozowski concluded that enhancing the local pool of growth factors, such as HGF and gastrin, at the ulcer site will likely become a new modality for ulcer treatment.

Platelet Derived Growth Factor (PDGF) As previously described, the mechanism underlying delayed ulcer healing induced by aspirin and indomethacin involves, if only in part, suppression of angiogenesis at the ulcer site. Accordingly, it would be of interest to examine whether or not PDGF could reverse the noxious effects of aspirin and indomethacin on delayed acetic acid ulcer healing in rats. Arceiz et al. ${ }^{121)}$ confirmed that both aspirin and indomehacin significantly delayed gastric ulcer healing, reducing ulcer contraction, mucosal regeneration, and cell proliferation. Both aspirin and indomethacin delayed the healing rate of gastric ulcers and reduced ulcer contraction, mucosal regeneration and cell proliferation. All these effects were completely reversed by oral treatment with PDGF-BB without affecting gastric acid secretion. Oral administration of PDGF accelerates ulcer healing and reverses the effects induced by NSAIDs on ulcer healing without affecting gastric secretion. Nonetheless, the detailed mechanism underlying such effects remains unknown. In particular, it is unclear whether or not angiogenesis was reversed by PDGF therapy.

Keratinocyte Growth Factor (KGF) Baatar et al. ${ }^{122)}$ reported that esophageal ulceration induced by local application of acetic acid activated KGF and its receptors in rats and suggested the potential role of $\mathrm{KGF}$ as a mediator of esophageal epithelial cell proliferation and ulcer healing.

5.3. Hormones It is rational to postulate that various hormones greatly contribute to ulcer healing. Recently, Machowska et al. ${ }^{123)}$ reported that testosterone significantly delayed healing of acetic acid ulcers in rats, probably by decreasing gastric mucosal blood flow and increasing production and release of the pro-inflammatory cytokine IL- $1 \beta$. In contrast, progesterone significantly enhanced ulcer healing, increasing gastric mucosal blood flow. It should be noted that testectomy improved gastric ulcer healing due to inhibition of gastric acid secretion and increased plasma gastrin levels.
In addition, Gunal et al. ${ }^{124)}$ found that estradiol improved the healing of acetic acid ulcers in rats. Bombesin, a gastrointestinal hormone, stimulates gastric acid secretion by releasing gastrin from antral $\mathrm{G}$ cells in both humans and animals. Gunal also demonstrated that bombesin significantly accelerated acetic acid ulcer healing in rats. Pre-treatment with CCK antagonists did not affect the positive healing effects of bombesin, suggesting that CCK receptors are not involved in the mechanism underlying enhanced ulcer healing. Guanl's studies did not support the notion that increased gastrin levels might exert a beneficial effect on ulcer healing. Reubi et $a l{ }^{125)}$ examined whether or not somatostatin receptors in the gastric mucosa are affected during the healing process of acetic acid ulcers in rats; somatostatin is a growth factor with multiple functions mediated by specific receptors. It was found that high affinity somatostatin receptors are present in the gastric mucosa in normal rats. In contrast, in healing ulcers, somatostatin receptors are almost non-existent at ulcer edges or ulcer scars even $84 \mathrm{~d}$ after ulcer induction. In contrast, the number of EGF receptors is increased in healing ulcers. Eeubi indicated that the persistent absence of somatostatin receptrors for several months after ulceration might be of pathophysiological significance for ulcer healing. Sun et $a l^{126)}$ examined plasma gastrin and somatostatin levels, as well as the number of $G$ and $D$ cells in the antrum and the $\mathrm{G} / \mathrm{D}$ ratio, in rats with acetic acid ulcers. Sun found increased gastrin levels and G/D ratios, as well as decreased somatostatin levels after ulceration; nonetheless, such changes gradually returned to normal as healing progressed.

5.4. Antibiotic Drugs Since $H$. pylori was found to exert a noxious effect on normal gastric mucosa, several antibiotic drugs are now prescribed upon detection of the bacteria. ${ }^{127)}$ We examined whether or not several antibiotic drugs affect the healing of acetic acid ulcers in $H$. pylori-infected rats and M. gerbils, as well as in non-infected animals. At first, we examined the effects of clarithromycin, amoxicillin, and metronidazole on ulcer healing in $H$. pylori-infected rats. As expected, all of the drugs potently eradicated the bacteria, yet clarithromycin alone significantly delayed ulcer healing in a dose-related manner. ${ }^{128)}$ Both amoxicillin and metronidazole had little or no effect on ulcer healing. Upon administration of clarithromycin, it was found that basal gastric acid secretion was markedly increased. It is also of interest that clarithromycin significantly delayed ulcer healing in non-infected rats as well. This finding suggests that the noxious effect of clarithromycin is not due to a toxin released from the eradicated $H$. pylori, but, rather, clarithromycin itself might have a deleterious effect on the mechanism underlying ulcer healing. Nonetheless, combined treatment of clarithromycin and acid pump inhibitors prevented delayed ulcer healing and resulted in enhancement of ulcer healing in comparison with administration of acid pump inhibitors alone. In M. gerbils, a 2-week administration of clarithromycin beginning 5 months after $H$. pylori infection not only significantly eradicated $H$. pylori, but also succeeded in preventing the development of gastric ulceration 6 months after infection. Clarithromycin administered to mice and M. gerbils with acetic acid ulcers markedly delayed spontaneous healing. In the $H$. pylori-infected animals, clarithromycin treatment prevented a delay in ulcer healing.

5.5. Miscellaneous Drugs and Endogenous Sub- 
stances Konturek et al. ${ }^{129)}$ examined the effects of pioglitazone, a peroxisome proliferator-activated receptor-gamma agent, on gastric ulcer healing, as well as the underlying mechanism for its effects. Konturek found that pioglitazone enhanced acetic acid ulcer healing, possibly by inducing hyperemia at the ulcer margins and inducing an anti-inflammatory effect, which included suppression of IL- $1 \beta$, TNF- $\alpha$, COX-2, and iNOS, and over-expression of HSP70. Activin $\mathrm{A}$, a homodimer of the activin/inhibin $\beta$ A subunit, is known to participate in cutaneous wound healing. Becker et al. ${ }^{130)}$ confirmed that activin A protein and mRNA expression was significantly increased in the bases and margins of acetic acid ulcers in rats. Becker thus suggested that activin A participates in gastric ulcer healing in a similar fashion as in cutaneous wound healing. Watanabe et al. ${ }^{131)}$ reported the effects of rebamipide on delayed acetic acid ulcer healing induced in M. gerbils by $H$. pylori infection. Rebamipide significantly prevented delay of ulcer healing by the bacteria. Watanabe suggested that the drug exerted its effect by modulating cell kinetics (apoptotic and proliferating cells) and inhibiting neutrophil infiltration (MPO activity). Brzozowski et al. ${ }^{132)}$ found that exogenous melatonin and L-tryptophan significantly accelerated acetic acid ulcer healing in rats, probably via interaction with MT2 receptors. The underlying mechanism appears to involve increases in gastric mucosal blood flow via COX-derived PGs, iNOS-derived NO, and CGRP released from sensory nerves. Konturek's group ${ }^{133)}$ also examined the role of exogenously administered leptin in ulcer healing. Konturek found that leptin significantly accelerated the healing of acetic acid ulcers by a mechanism involving up-regulation of TGF $\alpha$ and increased production of NO due to up-regulation of cNOS and iNOS in the ulcer area. Li et $a l^{134)}$ reported that subcutaneously or orally administered unfractionated and low molecular heparin accelerated the healing of acetic acid ulcers in rats. The enhanced ulcer healing effect of heparin was accompanied by a significant increase in mucosal regeneration and proliferation, angiogenesis, and mucus secretion, without affecting bleeding time. Kurinets and Lichtenberger et al. ${ }^{135)}$ provided a new hypothesis, stating that the mechanism underlying the damaging effect of aspirin for the gastric mucosa might be impairment of the phospholipid layer on the mucosal surface, leading to penetration of hydrochloric acid into the mucosa. Given this hypothesis, the effects of aspirin alone and aspirin chemically associated with phosphatidilcholine (aspirin-PC) on the healing of acetic acid ulcers were examined in rats. Aspirin-PC resulted in enhanced ulcer healing compared with rats treated with aspirin alone. They concluded that the protective hydrophobic lining of the stomach is maintained with PC-aspirin, thereby allowing ulcer healing of the tissue to proceed.

Motilva et al. ${ }^{136)}$ examined the role of oxygen-derived free radicals by measuring MPO as an index of leukocyte infiltration. MPO activity was significantly increased 7 and $14 \mathrm{~d}$ after acetic acid ulcer induction. Intraperitoneal administration of hydroxyurea significantly decreased the severity of gastric ulcers and neutrophil infiltration into the gastric lesions. In contrast, allopurinol did not produce any beneficial effect on either the macroscopic and histological appearance or vascular permeability. Such findings suggest that oxygenderived free radicals may contribute to the development of gastric ulcers and that such radicals were generated from neutrophils, but not from the xanthine oxidase pathway.

5.6. Gene Therapy Most gastric ulcers heal spontaneously or upon treatment with currently available anti-ulcer drugs, to include acid pump inhibitors and sucralfate. Nonetheless, there are intractable ulcers that are refractory to drug therapy. It has been reported that ulcers with bases that adhere to or penetrate into surrounding organs due to free or confined perforation are strongly resistant to drug therapy. In such cases, surgical intervention, to include highly selective vagotomy, is necessary to treat such ulcers. In recent years, gene therapy has become popular as a potential treatment for various diseases that cannot be managed with routine drug treatment. Since the important role of various growth factors was recently elucidated, gene therapy was incorporated into the medicinal treatment of peptic ulcers. Jones et al. ${ }^{137)}$ is the first to perform a series of experiments in an attempt to examine the effects of gene therapy on ulcer treatment, confirming its utility in animal experiments. Jones locally injected plasmid-encoding cDNA of rhVEGF, rhAng (angiopoietin)-1, or both into acetic acid ulcers in rats. Each plasmid caused increased neo-vascularization and accelerated ulcer healing. Combined treatment with two plasmids resulted in formation of more mature vessels and more complete restoration of gastric glandular structures within the ulcer scar. Serum response factor (SRF) has been found to regulate transcription of immediate early genes and muscle genes. SRF is known to be upregulated in human gastric ulcers and was experimentally confirmed to be upregulated in rats with acetic acid ulcers. ${ }^{138)}$ Local injection of SRF-expression plasmid into the ulcer base significantly enhanced ulcer healing, promoted re-epithelization, and enhanced muscle restoration. Such findings strongly suggest that activation of SRF is an important component of ulcer healing. In addition, Baatar et al. ${ }^{139)}$ suggested that, in the case of esophageal ulcers, gene therapy is potentially very feasible. Indeed, injection of cDNA-encoding VEGF significantly enhanced angiogenesis and accelerated esophageal ulcer healing in rats.

\section{SURGICAL TREATMENT}

Omental implantation is a surgical procedure in which a perforated gastric or duodenal ulcer is repaired by drawing and implanting a portion of the omentum into the digestive tract to accelerate ulcer healing and inhibit ulcer relapse. Matoba et al. ${ }^{140)}$ evaluated the effects of omental implantation for perforated gastric ulcers using an acetic acid ulcer model in rats. Greater anti-inflammatory, angiogenic activity, and accelerated collagen synthesis were seen after omental implantation. Moreover, bFGF-mediated angiogenesis and TGF- $\beta_{1}$ activity within and around the omentum were also found in the implantation group. It was noted that omental implantation accelerated acetic acid ulcer healing and inhibited ulcer relapse. We also confirmed the effects of omental implantation in rats with acetic acid ulcers. A large amount of omentum was drawn into the stomach through the artificially perforated acetic acid ulcers and implanted. Ten days later, the omentum in the stomach was completely digested away and the ulcer size was extensively diminished. Such findings confirm the usefulness of omental implantation for enhancing ulcer healing.

Tsukimi et al. ${ }^{19)}$ demonstrated with the kissing ulcer 
model that unilateral (anterior) vagotomy significantly enhanced anterior wall ulcer healing in rats, i.e., the ulcer induced in the posterior wall was not affected. They postulated that the mechanism of enhanced ulcer healing by vagotomy might be partly due to inhibited acid secretion and partly due to reduced expansion of the stomach on the anterior side in response to food ingestion, resulting in reduced exposure of the ulcerated area to acid and pepsin.

\section{MECHANISMS OF ULCER HEALING}

7.1. Ulcer Base Contraction Various factors are involved in ulcer healing. In particular, contraction of the ulcerated area, re-epithelization over the ulcer base, and formation of granulation tissue around the ulcerated area represent the principal steps in healing. Contraction of the wounded margin is requisite for wound healing. Even in the healing of gastric ulcers, contraction of the ulcer area, as well as inward migration of newly formed epithelium from the ulcer margin, is considered to represent a crucial event. Majno et al. ${ }^{141)}$ reported that the granuloma pouch tissue contracts just like smooth muscle in response to 5-hydroxytryptamine, vasopressin, bradykinin, or $\mathrm{PGF}_{1 \alpha}$ presumably since myofibroblasts are present in the granulation tissue. Nakamura et al. ${ }^{142)}$ noted the presence of myofibroblasts at the base of acetic acid-induced gastric ulcers in rats. In a histologic study, we found that the length of the rupture of the muscularis mucosae was significantly longer in the groups treated with indomethacin and prednisolone compared with controls. Such results suggest that the drugs might partly prevent contraction of the connective tissue at the ulcer base, probably by counteracting the action of released chemical mediators or preventing the release of such mediators. Most anti-inflammatory drugs suppress PG synthesis by inhibiting COX and phospholipase $\mathrm{A}_{2}$ activity or the release of PGs. Accordingly, it is most likely that indomethacin and prednisolone interfere with contraction of the ulcer area as a result of a reduction in endogenous PGs. In addition, the well-developed fibrosis observed in the indomethacin-treated group might decrease the elasticity of the tissue, preventing contraction of the ulcer area. In the prednisolone-treated group, however, there exists the possibility that the ulcer area could not contract due to the fact that formation of connective tissue, which involves myofibroblasts, was reduced by the drug. We suggest that the mechanisms by which indomethacin and prednisolone delay ulcer healing differ; nonetheless, a common mechanism related to prevention of contraction of connective tissue at the ulcer base can be inferred. Together with Tsukimi, ${ }^{143)}$ we examined the effects of a liquid versus solid diet on the healing of acetic acid ulcers in rats. The liquid diet significantly enhanced ulcer healing in both early $(1-10 \mathrm{~d})$ and late $(10$ $20 \mathrm{~d})$ phases. As expected, we confirmed that the stomach administered a liquid diet alone did not dilate compared with the stomach administered a solid diet. The mechanism by which unilateral vagotomy accelerates the healing of ulcers on the denervated side appears to relate to the inhibition of both gastric acid secretion and gastric relaxation.

7.2. Epithelial Regeneration and Mucus Secretion In conjunction with contraction of the ulcerated area, regenerated epithelial cells migrate from the margins of the ulcerated area to cover the surface of the ulcer base. In the case of weak contraction, regardless of the etiology, ulcer healing will be delayed, as re-epithelization of a larger ulcerated surface will require additional time. Various substances are postulated to enhance formation of new epithelium. Hayashida et $a l .{ }^{144)}$ reported that using monoclonal antibodies, sialomucin was observed in regenerating epithelia during the healing stage of acetic acid ulcers.

7.3. Angiogenesis Granulation tissue develops at the ulcerated area and becomes the base of the recovering ulcer, consisting of connective tissue (collagen) and epithelium, which subsequently develops into vessels (angiogenesis). Needless to say, angiogenesis is one of the most important factors for ulcer healing, as it allows the supply of nutrients and oxygen to the damaged area to enhance its healing. The Tie-2 receptor tyrosine kinases and ligands, angiopoietin (Ang)-1 and -2 are known to play a crucial role in vascular development. Sekine's group ${ }^{145,146)}$ confirmed the expression of Tie- 2 and Ang-1 and - 2 mRNA in the base of acetic acid ulcers in rats. With Western blot analysis, it was demonstrated that Tie-2 expression reached a maximum on the $3 \mathrm{rd}$ to 5th day after ulceration, decreasing thereafter. Immunohistochemically, Tie-2 was found to be constitutively expressed in the endothelial cells of pre-existing vessels in the gastric wall, while Tie- 2 expression was increased in new capillaries of the ulcer base. Sekine indicated that Tie-2 and Ang-1 and -2 play a beneficial role for angiogenesis in the early phase of ulcer healing. In addition, Sekine demonstrated that rhIL-11 enhanced the Tie- 2 expression in the base and margins of acetic acid ulcers in rats. Such findings suggest that IL-11 could partly accelerate ulcer healing by up-regulating Tie-2 expression and thus promote angiogenesis. Indeed, Wen et $a l{ }^{147)}$ confirmed that subcutaneously administered IL-11 significantly enhanced acetic acid ulcer healing in rats. The mechanism underlying such a finding involves induction of mucosal and muscular cell proliferation, as determined by measuring proliferating cell nuclear antigen expression and 1L-11 $\alpha$ receptor expression in the gastric tissue.

Accordingly, it is rational to postulate that stimulation of angiogenesis enhances ulcer healing and inhibition of angiogenesis delays ulcer healing. Indeed, arachidonic acid (a prostaglandin precursor) and 16, 16 dimethyl $\mathrm{PGE}_{2}$, known as potent ulcer healing compounds, significantly stimulate angiogenesis in injured gastric mucosa. Exogenously-administered growth factors, such as EGF, bFGF, and VEGF have been found to further enhance ulcer healing, most probably through stimulation of either angiogenesis or proliferation of the extracellular matrix, which includes collagen in the ulcer base.

In contrast, it has been repeatedly confirmed that indomethacin significantly suppressed development of microvasculature (angiogenesis) in granulation tissue at ulcer bases. Accordingly, it is possible that the mechanism underlying delayed ulcer healing by indomethacin is reduction of PG levels in the ulcerated area and/or interference with other factors (bFGF, PDGF, TGF, etc.) that stimulate angiogenesis. With histological analysis, it was found that mucosal regeneration, maturation of the ulcer base, and angiogenesis in the base were significantly prevented by NS-398 (a selective COX-2 inhibitor) and indomethacin. It should be noted that the inhibitory effect of NS-398 was weaker than that of indomethacin. ${ }^{148)}$ In addition, Guo et al. ${ }^{149)}$ also demonstrated 
that rofecoxib, a highly selective COX-2 inhibitor, significantly increased the size of acetic acid ulcers in rats and decreased the amount of microvasculature, bFGF protein expression, and $\mathrm{PGE}_{2}$ levels. In our laboratory, the effects of p38, a MAP-kinase family compound, on the healing of acetic acid ulcers were examined in rats. ${ }^{150)}$ It is of interest that FR167653, a MAP-kinase inhibitor, significantly delayed ulcer healing and suppressed angiogenesis in the ulcer base. In addition, the production of IL- $1 \beta$, TNF $\alpha, \mathrm{PGE}_{2}$, and $\mathrm{NO} x$ at the ulcer margins was significantly reduced with FR167653 treatment. Such findings led to the conclusion that p38 activated by ulceration might be involved in the ulcer healing process.

7.4. Matrix Formation Needless to say, the quality of ulcer repair depends on the stability of the damaged tissue, which thus strongly affects the future relapse rate. Shahin et al. ${ }^{151)}$ examined extracellular matrix remodeling during acetic acid ulcer healing in rats for up to $30 \mathrm{~d}$ after ulceration. The temporo-spatial expression of the fibrillar and basement membrane collagens (types I, III, and IV), the collagenase MMP-2, as well as its inhibitor TIMP-1, were studied before and after oral bFGF administration. Shahin concluded that the early onset and long duration of collagen expression indicates the component's central structural and functional role in gastric ulcer healing. MMP-2 appears to be involved in both active ulceration and extracellular matrix remodeling. It is well known that fibronectin is important for wound healing via cell proliferation, adhesion, differentiation, and matrix formation. Tominaga et al. ${ }^{152)}$ examined the expression and localization of full-length fibronectin, as well as EIIIA- and EIIIB-fibronectin, in gastric tissue during acetic acid ulcer healing in rats. Full-length fibronectin mRNA levels were increased from days 3 to 32 and returned to control levels on day 60. Fibronectin predominantly localized at the mesenchyme around the infiltrating inflammatory cells in the granulation tissue and the basement membranes of the nonproliferating epithelial cells that were regenerating at the ulcer margins. Tominaga suggested that fibronectin plays an important role in gastric ulcer healing, mainly via enhancing migration of mesenchymal and epithelial cells. Ohmiya et al. ${ }^{153)}$ examined the kinetics and collagenolytic role of eosinophils in acetic acid ulcers in rats. It was found that eosinophils that express human metaloproteinase 1 infiltrated granulation tissue of open ulcers by 40 to $180 \mathrm{~d}$ after ulceration. Azan staining showed dispersed collagen fibers around infiltrating eosinophils. Accordingly, Ohmiya suggested that eosinophils may play a role in tissue remodeling and deterioration of ulceration.

\subsection{Factors Affecting Ulcer Healing}

Nitric Oxide (NO) NO dilates vascular smooth muscle to increase blood flow to various organs. Accordingly, it is readily postulated that if NO production is inhibited, ulcer healing would be delayed. Konturek et al. ${ }^{154)}$ reported the effects of $\mathrm{NO}$ synthase inhibitors, $\mathrm{N}^{\mathrm{G}}$-nitro-L-arginine and $\mathrm{N}^{\mathrm{G}}$ mono-methyl-L-arginine, and exogenous NO donors, such as glyceryl trinitrate, on the healing of acetic acid ulcers in rats. The inhibitors of NO synthase significantly delayed ulcer healing, reduced gastric mucosal blood flow at the ulcer margins, and decreased the number of capillaries in the granulation tissue at the ulcer bed. These effects were partly antagonized with glyceryl trinitrate and L-argine. Such data strongly suggest that endogenous NO plays an important role in maintenance of blood flow around ulcers, in angiogenesis in the granulation tissue, and in gastric ulcer healing.

Brzozowski et al. ${ }^{155)}$ determined the effects of L-arginine on acetic acid ulcer healing in rats. Orally administered Larginine significantly accelerated ulcer healing in a dose-related manner, while D-arginine was not effective. The enhanced ulcer healing was accompanied by a marked increase in gastric mucosal blood flow at the ulcer margins, as well as increased serum gastrin levels, mucosal DNA synthesis, and angiogenesis in the granulation tissue in the ulcer bed. Similar responses were observed with gylceryl trinitrate, an NO exogenous supplier. It is of interest that treatment with $N^{\mathrm{G}}$ nitro-L-arginine, an inhibitor of NO synthase, delayed ulcer healing and reduced mucosal blood flow, angiogenesis, and gastrin levels. In addition, inhibition of polyamine biosynthesis completely abolished the various effects of L-arginine on rats with ulcers. Brzozowski concluded that the ulcer healing effects of L-arginine are possibly related to NO, gastrin, and polyamines. As expected, repeated administration of NO synthase (NOS) inhibitor ${ }^{156}$ ) resulted in significantly delayed healing of Type 1 acetic acid ulcers in rats. Furthermore, Larginin clearly reversed the effects of NOS inhibitor on ulcer healing. Such results suggest that NO is one of the factors involved in ulcer healing, most probably by enhancing blood flow to the ulcerated area. Recently, Guo et al. ${ }^{157)}$ reported that high iNOS expression and activity were found in gastric ulcer bases in rats 1 to $3 \mathrm{~d}$ after ulceration. The maximum expression of the enzymes heme oxygenase-1 (HO-1) and COX-2, as well as the activity level of HO-1 and COX-2 lagged behind that of iNOS and remained at high levels during the healing phase. Guo concluded that iNOS may contribute to ulcer formation, while $\mathrm{HO}-1$ and $\mathrm{COX}-2$ may promote ulcer healing. Tatemichi et al. ${ }^{158)}$ confirmed iNOS expression at ulcer bases in mice and rats and reiterated the compound's important role for ulcer healing. It should be noted that in iNOS deficient mice, gastric ulcers induced by acetic acid were larger and exhibited more severe inflammation around the ulcer area during the healing process.

Capsaicin Since its isolation from red peppers, accumulating evidence indicates that capsaicin-sensitive afferent fibers play an important role in gastroprotection and gastric ulcer healing. Nonetheless, inconsistent results concerning the effects of capsaicin have been reported. Tramontana et $a l .{ }^{159)}$ examined the influence of capsaicin-sensitive afferent fibers on acetic acid ulcer healing in rats. Tramontana demonstrated that afferent nerve ablation by capsaicin both produced a significant increase in the ulcer area 1 and 2 weeks after ulceration and delayed ulcer healing. The mechanisms remain unknown. Konturek et al. ${ }^{160)}$ also demonstrated that capsaicin significantly delayed acetic acid ulcer healing in rats, possibly by significantly decreasing gastric mucosal blood flow and significant increasing gastric mucosal gene expression of IL-1 $\beta$ and TNF $\alpha$. In addition, Ma et al. ${ }^{161)}$ clearly demonstrated that although ulcer induction extensively elevated EGF levels in salivary glands and serum, capsaicin treatment completely inhibited such changes. Furthermore, capsaicin significantly increased ulcer size, but decreased gastric mucosal cell proliferation at the ulcer margins, angiogenesis in the granulation tissue, and gastric mucus quantity. Ma concluded that stimulation of EGF ex- 
pression in salivary glands and serum may represent one of the mechanisms by which capsaicin-sensitive nerves contribute to ulcer healing in the stomach. We also confirmed that orally administered capsaicin, before and after acetic acid ulcer induction in rats for $7 \mathrm{~d}$, resulted in development of large ulcers in all rats compared with non-treated rats (unpublished data). In contrast, Kang et al. ${ }^{162)}$ reported that capsaicin significantly promoted healing of acetic acid ulcers in rat stomachs due to its gastric hyperemic effect. It is of interest that treatment with exogenous leptin significantly accelerated Type 2 ulcer healing, increased gastric mucosal blood flow at the ulcer margins and upregulated mRNA for iNOS. Since such effects were attenuated by capsaicin-mediated deactivation of sensory neurons, the ulcer healing effect of leptin may involve sensory afferent neurons.

Circulating Gastrin Proton pump inhibitors (PPI), such as omeprazole, lansoprazole, pantoprazole, and rabeprazole, enhance gastric and duodenal ulcer healing in humans and animals via their potent anti-secretory effects. ${ }^{163)}$ With prolonged treatment in rats, such drugs invariably result in marked hypergastrinemia due to sustained inhibition of gastric acid secretion. ${ }^{164-173)}$ It is well known that gastrin has a trophic effect on gastrointestinal mucosal cells, to include surface epithelial and ECL cells. In recent years, the underlying mechanism of gastrin's trophic effect has been elucidated at the molecular level. Fukui et al. ${ }^{174)}$ suggested that the Reg protein, which is released from ECL cells in response to gastrin, is involved in stimulation of the proliferative zone. Miyazaki et al. ${ }^{175)}$ reported that gastrin stimulates expression of heparin-binding EGF-like growth factor (HB-EGF) mRNA. Moreover, Miyazaki also demonstrated that such an effect of gastrin is partially inhibited by anti-rat HB-EGF serum and completely blocked by AG1478, an EGF receptorspecific tyrphostin. Regardless of the exact mechanism underlying gastrin's action, it has been postulated that hypergastrinemia resulting from PPI greatly contributes to ulcer healing due to gastrin's trophic effect. Indeed, Ito et al. ${ }^{176)}$ and $\mathrm{Li}$ and Helander ${ }^{177)}$ demonstrated increased epithelial proliferation around acetic acid ulcers, as well as hypergastrinemia, after omeprazole or exogenous gastrin treatment. Based on such data, it was generally suggested that such stimulated epithelial proliferation in response to increased gastrin levels is directly related to the enhanced ulcer healing that was observed. Brzozowski et al. ${ }^{178)}$ found that melatonin and its precursor L-tryptophan significantly enhanced acetic acid ulcer healing in rats. Gastrin levels were significantly increased upon exposure to the two compounds. Accordingly, Brzozowski postulated that gastrin might be involved in the mechanism underlying the action of melatonin and its precursor.

In an attempt to clarify the role of gastrin on ulcer healing, we examined the effects of a somatostatin analog (SDZ CO611) and selective gastrin/CCK2 antagonists, such as YM022 and S-0509, on the ulcer healing effect of omeprazole. We found that concurrent administration of a SDZ CO-611 with omeprazole significantly suppressed hypergastrinemia in rats with acetic acid ulcers. ${ }^{179)}$ The ability of omeprazole to positively impact ulcer healing was not affected. In addition, we demonstrated that the ulcer healing effects of omeprazole were not affected by simultaneous treatment with YM022 or S-0509. Such results strongly suggest that the trophic action of circulating gastrin released in response to omeprazole does not play a role in the mechanism underlying enhanced ulcer healing by omeprazole. We conclude that omeprazole and gastrin receptor antagonists, such as YM022 and S-0509, might contribute to gastric ulcer healing via their anti-secretory effects, but not through production of a concomitant hypergastrinemic state.

Endogenous PGs Since PGs were discovered in the gastric mucosa, their role in regulating gastric acid secretion, maintaining gastric mucosal integrity, and ulcer healing has been extensively studied. One of the many reasons that PGs appear to be important for the pathophysiology of the gastrointestinal tract is that various NSAIDs induce gastric mucosal damage and delay ulcer healing. In addition, exogenously administered PGs significantly prevent development of mucosal damage, as well as delay in ulcer healing. ${ }^{179)} \mathrm{We}$ confirmed that the delayed healing caused by indomethacin was clearly prevented by concurrent treatment with $\mathrm{PGE}_{2}$ for a period of 2 or 4 weeks. ${ }^{38)}$ Mizuno et al. ${ }^{180)}$ reported with acetic acid ulcers induced in mice that generation of PGs was significantly increased in the ulcerated area through the action of COX-2. The inhibition of the enzyme with NS-398 resulted in a delay of ulcer healing. Mizuno suggested that PGs generated by COX-2 are a prerequisite for ulcer healing. We also examined the localization and regulation of COX-2 in ulcerated areas of rat stomachs. We found that during the initial week after ulceration, $\mathrm{PGE}_{2}$ generation was approximately 3 fold in the ulcer base compared with intact areas. Paralleling the generation of PG, COX-2 was also highly expressed in the ulcer base during the period, yet gradually returned to normal levels, i.e. nearly zero. Both indomethacin and NS-398 resulted in a significant delay in ulcer healing, as well as a reduction of $\mathrm{PGE}_{2}$ generation in the ulcerated area in a dose-related manner. It is of interest that mRNA for cytokines such as IL- $1 \beta$, TNF- $\alpha$, and TGF- $\beta_{1}$ was clearly expressed in the ulcerated area. We found that COX-2 mRNA expression is regulated positively by IL- $1 \beta$ or TNF- $\alpha$, but negatively by TGF- $\beta_{1}$. It is quite apparent that COX- 2 expression in the ulcer area is regulated by several cytokines and plays a crucial role in acetic acid ulcer healing.

Heat Shock Protein (HSP) HSP is generally induced when cells are subjected to noxious stimuli. Guo et al. ${ }^{181)} \mathrm{de}-$ termined the expression and immusnolocalization of iHSP70, HSP47, and HSP32 in gastric tissue samples obtained from ulcer bases, ulcer margins, and non-ulcerated areas in rats; the kissing ulcer model was used for the experiments. Guo concluded that HSP32 appears to act as an inflammatory defensive factor, while HSP47 acts as a collagen-specific molecular chaperone contributing to gastric ulcer healing; the role of iHSP70 in ulcer healing remains undefined. Our group ${ }^{182)}$ examined the effects of omeprazole and indomethacin on the healing of kissing ulcers and mucosal HSP expression in rats. We found that, while enhanced expression of HSP70 is involved in acceleration of ulcer healing, over-expression of HSP47 is involved in delayed ulcer healing.

\section{MISCELLANEOUS STUDIES WITH ACETIC ACID ULCERS}

It is empirically known that changes in visceral sensation contribute to development of dyspepsia. Lamb et al. ${ }^{183)}$ ex- 


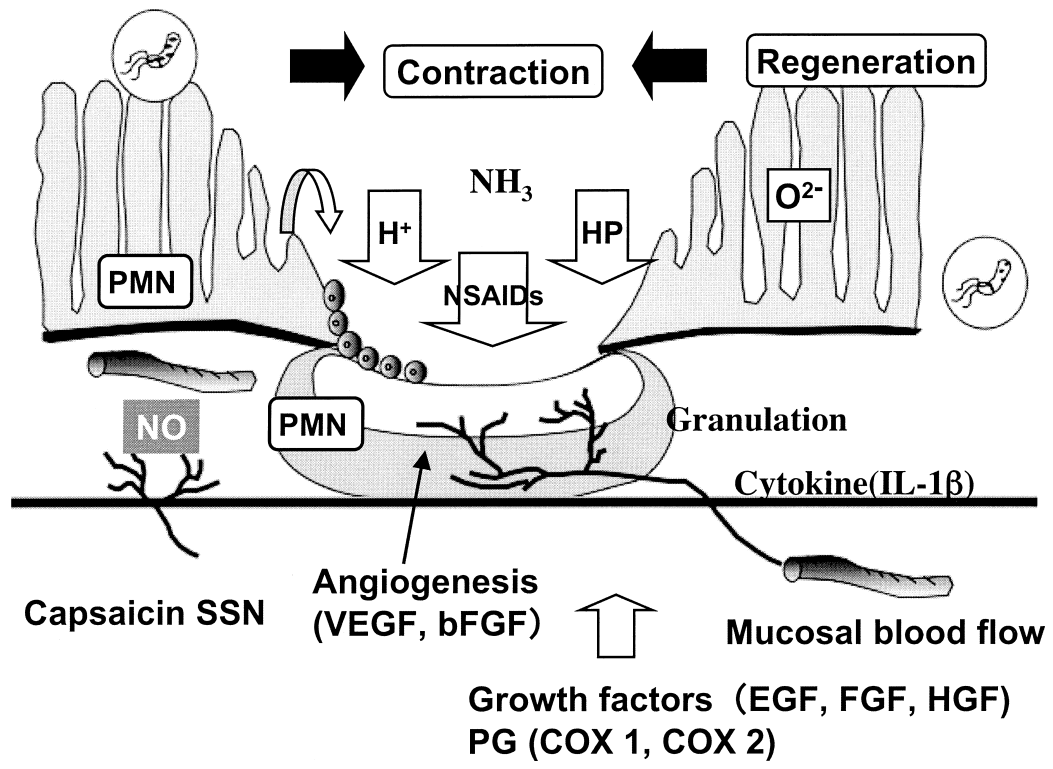

Fig. 11. Schematic Drawing of Healing Process of Acetic Acid Ulcer Induced in Rat Stomach

PMN, polymorphonuclear cells; HP, Helicobacter pylori; NO, nitric oxide; Capsaicin SSN, Capsaicin sensory sensitive neurons.

amined whether or not the presence of gastric inflammation, such as acute gastritis and chronic ulcers (Type 3 acetic acid ulcers) triggers hypersensitivity to acid in awake rats. Lamb confirmed that such inflammation enhanced the visceromotor response to intragastric acid through the changes of the acromiotrapezius muscle. Mei et al. ${ }^{184)}$ examined tissue infiltration and activation of eosinophils in ulcer margins at different time points after acetic acid ulcer induction in rats. For that purpose, eosinophil peroxidase staining and transmission electron microscopy were used. Mei concluded that there was marked infiltration and signs of activation of eosinophils together with macrophages at the margins of newly formed ulcers. In our laboratory, a neutrophil anti-chemotactic substance was found in the gastric mucosa of rats with acetic acid ulcers. Accordingly, the role of the substance was examined in relation to the mechanism by which indomethacin delays ulcer healing in rats. We found that the ulcerated tissue exhibited reduced anti-chemotactic activity and increased chemotactic acitivity, resulting in persistence of neutrophil infiltration. Naito et $a l^{185)}$ suggested that scavenging hydroxyl radicals with dimethyl sulfoxide or inhibiting xanthine oxidase with allopurinol enhances the quality, but not the speed, of acetic acid ulcer healing in rats.

\section{CONCLUSION}

Over the years, an increasing number of studies involving the above acetic acid models have been undertaken to strive towards both clarification of the mechanisms underlying ulcer healing and discovery of additional anti-ulcer pharmaceuticals (Fig. 11). Utilizing molecular biology techniques, to include gene therapy, it is now possible to more precisely analyze the mechanisms underlying ulcer healing, expediting discovery of more effective ulcer therapy. Given the above, we conclude that acetic acid ulcer models appear to be incredibly useful for pathophysiological and pharmacological studies of peptic ulcers. With the use of acetic acid ulcer models, a new anti-ulcer drug that prevents ulcer relapse, as well as enhances ulcer healing, could potentially be developed.

Acknowledgements The authors wish to thank to Professors Keijiro Takagi, Yutaka Kasuya, Yasuo Gomi, Issei Takayanagi, Hitoshi Kato, Kazuo Watanabe, and Koji Takeuchi, as well as Drs. Yasuo Ishii, Yasuhiro Tsukimi, and Takeshi Nishino, for their valuable advice and collaboration in achieving the above works. We also extend our sincere thanks to Professors Hideomi Fukuda and Tsutomu Kameyama for their encouragement. Special thanks go to Dr. Christopher Hurt (Department of Radiology, University of Washington) for manuscript review.

\section{REFERENCES AND NOTES}

1) Present address: Department of Pharmacology, Faculty of Pharmacy, Doshisha Women's College of Liberal Arts; Kodo, Kyotanabe, Kyoto 610-0395, Japan.

2) Shay H., Komarov S. A., Fels S. S., Meranze D., Gruenstein M., Siplet H., Gastroenterology, 4, 43-61 (1945).

3) Takagi K., Kasuya Y., Watanabe K., Chem. Pharm. Bull., 12, 465472 (1964).

4) Takagi K., Okabe S., Jpn. J. Pharmacol., 18, 9-18 (1968).

5) Takagi K., Okabe S., Saziki R., Jpn. J. Pharmacol., 19, 418-426 (1969).

6) Okabe S., Pfeiffer C. J., Am. J. Dig. Dis., 17, 619-629 (1972).

7) Robert A., Selye H., Ann. Endocrinol., 13, 845-848 (1952).

8) Okabe S., Roth J. L. A., Pfeiffer C. J., Am. J. Dig. Dis., 16, 277-284 (1971).

9) Tarnawski A., Hollandeer D., Krause W. J., Dabros W., Stachura J., Gergely H., J. Clin. Gastroenterol., 12, S139-S147 (1990).

10) Bui H., H. X., Lee C. Y., del Rosario A., Abdulla M., Ballouk F., Khan M., Sheehan C., Ross J. S., Exp. Mol. Pathol., 59, 136-154 (1993).

11) Keto Y., Ebata M., Tomita K., Okabe S., Dig. Dis. Sci., 47, 837-849 (2002).

12) Okabe S., Roth J. L. A., Pfeiffer C. J., Experientia, 27, 146-148 (1971).

13) Skarstein A., Svanes K., Scand. J. Gastroenterol., 10, 339-345 
(1975).

14) Skartstein A., Scand. J. Gatroenterol., 14, 905-911 (1979).

15) Sugawa C., Lucas C. E., Walt A. J., Surgery, 70, 590-595 (1971).

16) Takeuchi K., Okabe S., Takagi K., Umeda N., Digestion, 16, 51-56 (1977).

17) Takeshita H., Kotani Y., Okabe S., Dig. Dis. Sci., 29, $841-847$ (1984).

18) Koga T., Shimada Y., Okazaki Y., Takahashi K., Sato K., Kikuchi I., Katsuta M., Iwata M., J. Microbiol. Immunol. Infect., 36, 218-222 (2003).

19) Tsukimi Y., Okabe S., Jpn. J. Pharmacol., 66, 105-114 (1994).

20) Tsukimi Y., Okabe S., J. Gastroent. Hepatol., 9, S60-S65 (1994).

21) Tsukimi Y., Okabe S., J. Gastroent. Hepatol., 11, 335-340 (1996).

22) Tsukimi Y., Okabe S., Jpn. J. Pharmacol., 68, 103-110 (1995).

23) Penney A. G., Andrews F. J., O’Brien P. E., Dig. Dis. Sci., 41, 18381844 (1996).

24) Amagase K., Okabe S., Inflammopharmacol., 10, 385-389 (2002).

25) Fukawa K., Kawano O., Misaki N., Uchida M., Irino O., Jpn. J. Pharmacol., 33, 175-179 (1983).

26) Uchida M., Kawano O., Misaki N., Saitoh K., Irino O., Chem. Pharm. Bull., 37, 805-807 (1989).

27) Arakawa T., Watanabe T., Fukuda T., Higuchi K., Takaishi O., Yamasaki K., Kobayashi K., Tarnawski A., Dig. Dis. Sci., 41, 20552061 (1996).

28) Okabe S., Saziki R., Takagi K., J. Appl. Physiol., 30, 793-796 (1971).

29) Skoryna S. C., Webster D. R., Kahn D. S., Gastroenterology, 34, $1-$ 10 (1958).

30) Phillips M. J., Skoryna S. C., Webster D. R., Kahn D. S., Canad. J. Surg., 4, 239-248 (1961).

31) Umehara S., Tabayashi T., Shibuya E., Med. Biol., 66, 7-10 (1963).

32) Kahn D. S., Phillips M. J., Skoryna S.C., Am. J. Pathol., 38, 73-87 (1961).

33) Hase S., Nakazawa S., Tsukamoto Y., Segawa K., Digestion, 42, $135-142$ (1989).

34) Kuwayama H., Matsuo Y., Eastwood G. L., Am. J. Med., 91, S15S19 (1991).

35) Amagase K., Yokota M., Okabe S., J. Physiol. Pharmacol., 54, 349360 (2003).

36) Luo J. C., Shin V. Y., Liu E. S., Ye Y. N., Wu W. K., So W. H., Chang F. Y., Cho C. H., Eur. J. Pharmacol., 485, 275-281 (2004).

37) Szelenyi I., Engler H., Herzog P., Postius S., Vergin H., Holtermuller K. H., Agents and Actions, 12, 180-182 (1982).

38) Szelenyi I., Postius S., Engler H., Agents and Actions, 13, 207-209 (1983).

39) Wang J. Y., Yamasaki S., Takeuchi K., Okabe S., Gastroenterology, 96, 393-402 (1989)

40) Hirose H., Takeuchi K., Okabe S., Gastroenterology, 100, 1259 1265 (1991).

41) Satoh H., Shino A., Sato F., Asano S., Murakami I., Inatomi N., Nagaya H., Kato K., Szabo S., Folkman J., Jpn. J. Pharmacol., 73, 5971 (1997)

42) Satoh H., Asano S., Maeda R., Murakami I., Inada I., Sato F., Shino A., Jpn. J. Pharmacol., 73, 229-241 (1997).

43) Hirose Y., Goto H., Arisawa T., Hase S., Niwa Y., Hayakawa T., Asai J., Tsukamoto Y., Digestion, 58, 332-339 (1997).

44) Lyons K. C., Andrews F. J., Comper W. D., O’Brien P. E., Dig. Dis. Sci., 42, 1755-1764 (1997).

45) Penney A. G., Malcontenti-Wilson C., O’Brien P. E., Andrews F. J., Dig. Dis. Sci., 40, 2684-2693 (1995).

46) Naito Y., Yoshikawa T., Matsuyama K., Nishimura S., Yagi N., Kondo M., Dig. Dis. Sci., 40, 2019-2021 (1995).

47) Okabe S., Takeuchi K., Honda K., Takagi K., Digestion, 14, 85-88 (1976).

48) Brzozowski T., Targosz A., Sliwowski Z., Kwiecien S., Drozdowicz D., Pajdo R., Konturek P. C., Brzozowski T., Pawlik M., Konturek S. J., Pawlik W. W., Hahn E. G., J. Physiol. Pharmacol., 55, 773-790 (2004).

49) Slomiany B. L., Slomiany A., IUBMB Life, 50, 391-395 (2000).

50) Ulaganathan M., Familari M., Yeomans N. D., Giraud A. S., Cook G. A., J. Gastroenterol. Hepatol., 16, 506-512 (2001).

51) Sanchez-Fidalgo S., Martin-Lacave I., Illanes M., Motilva V., Eur. J. Pharmacol., 505, 187-194 (2004).

52) Sanchez-Fidalgo S., Martin-Lacave I., Illanes M., Bruseghini L., Es- teras A., Motilva V., Histol. Histopathol., 20, 437-447 (2005).

53) Berenguer B., Alarcon de la L. C., Moreno F. J., Martin M. J., Eur. J. Pharmacol., 442, 125-135 (2002).

54) Slomiany B. L., Slomiany A., Inflammopharmacol., 11, 237-248 (2003).

55) Laudanno O. M., Cesolari J. A., Esnarriaga J., Rista L., Piombo G., Maglione C., Aramberry L., Sambrano J., Godoy A., Rocaspana A., Dig. Dis. Sci., 46, 779-784 (2001).

56) Akiba Y., Nakamura M., Mori M., Suzuki H., Oda M., Kimura H., Miura S., Tsuchiya M., Ishii H., J. Clin. Gastroenterol., 27, S64S73 (1998).

57) Araki H., Komoike Y., Matsumoto M., Tanaka A., Takeuchi K., Digestion, 66, 145-153 (2002).

58) Baatar D., Jones M. K., Pai R., Kawanaka H., Szabo I. L., Moon W. S., Kitano S., Tarnawski A. S., Am. J. Pathol., 160, 963-972 (2002).

59) Shen X., Zhonghua Yi Xue Za Zhi, 81, 1380-1383 (2001).

60) Brzozowski T., Konturek P. C., Konturek S. J., Sliwowski Z., Pajdo R., Drozdowicz D., Ptak A., Hahn E. G., Microsc. Res. Tech., 53, $343-353$ (2001).

61) Ma L., Elliott S. N., Cirino G., Buret A., Ignarro L. J., Wallace J. L., Proc. Natl. Acad. Sci. U.S.A., 98, 6470-6475 (2001).

62) Harsch I. A., Brzozowski T., Bazela K., Konturek S. J., Kukharsky V., Pawlik T., Pawlowski E., Hahn E. G., Konturek P. C., Eur. J. Pharmacol., 481, 249-260 (2003).

63) Iwata F., Leung F. W., Scand. J. Gastroenterol., 30, 315-321 (1995).

64) Shin V. Y., Liu E. S., Koo M. W., Wang J. Y., Matsui H., Cho C. H., Exp. Biol. Med., 227, 114-124 (2002).

65) Marshall B. J., Warren J. R., Lancet, 1, 1273-1275 (1983).

66) Bui H. X., del Rosario A., Sonbati H., Lee C. Y., George M., Ross J. S., Exp. Mol. Pathol., 55, 261-268 (1991).

67) Li T., Okabe S., J. Gastroenterol. Hepatol., 13, S185-S189 (1998).

68) Konturek P. C., Brzozowski T., Konturek S. J., Stachura J., Karczewska E., Pajdo R., Ghiara P., Hahn E. G., Aliment. Pharmacol. Ther, 13, 333-346 (1996).

69) Brzozowski T., Konturek P. C., Konturek S. J., Kwiecien S., Pajdo R., Karczewska E., Stachura J., Hahn E. G., Digestion, 60, 22-33 (1999).

70) Gunawan E., Tsuji S., Tsujii M., Kimura A., Sun W. H., Sawaoka H., Sasayama Y., Sasaki Y., Kawano S., Hori M., J. Gastroenterol. Hepatol., 17, 960-965 (2002).

71) Hata M., Yamazaki Y., Ueda T., Kato T., Kohli Y., Fujiki N., Eur. J. Histochem., 38, 41-52 (1994).

72) Kodama R., Fujioka T., Shuto R., Kubota T., Nasu M., J. Gastroenterol. Hepatol., 11, 1097-102 (1996).

73) Hirayama F., Takagi S., Kusuhara H., Iwao E., Yokoyama Y., Ikeda Y., J. Gastroenterol., 31, 755-757 (1996).

74) Matsumoto S., Washizuka Y., Matsumoto Y., Tawara S., Ikeda F., Yokota Y., Karita M., J. Med. Microbiol., 46, 391-397 (1997).

75) Takahashi S., Keto Y., Fujita H., Muramatsu H., Nishino T., Okabe S., Dig. Dis. Sci., 43, 754-765 (1998).

76) Black J., Duncan W. A. M., Durant C. J., Ganellin C. R., Parsons E. M., Nature (London), 236, 385-390 (1972).

77) Ganser A. L., Forte J. G., Biochim. Biophys. Acta, 307, 169-180 (1973).

78) Fellenius E., Elander B., Wallmark B., Helander H. F., Berglindh T., Am. J. Physiol., 243, G505-G510 (1981).

79) Rajendra S., Mulcahy H., Patchett S., Kumar P., Dig. Dis. Sci., 49, 1634-1640 (2004).

80) Tsuchida T., Tsukamoto Y., Segawa K., Goto H., Hase S., Digestion, 47, 8-14 (1990).

81) Yamamoto O., Okada Y., Okabe S., Dig. Dis. Sci., 29, $394-401$ (1984).

82) Wang J. Y., Nagai H., Okabe S., Jpn. J. Pharmacol., 54, 82-85 (1990).

83) Satoh H., Inatomi N., Nagaya H., Inada I., Nohara A., Nakamura N., Maki Y., J. Pharmacol. Exp. Ther, 248, 806-815 (1989).

84) Takeuchi K., Konaka A., Nishijima M., Kato S., Yasuhiro T., J. Gastroenterol. Hepatol., 14, 251-257 (1999).

85) Ishihara M., Ito M., Eur. J. Pharmacol., 444, 209-215 (2002).

86) Kuwayama H., Matsuo Y., Eastwood G. L., Am. J. Med., 91, 15S19S (1991).

87) Ramaswamy S., Life Sci., 70, 17-24 (2001).

88) Ogihara Y., Okabe S., J. Physiol. Pharmacol., 44, 109-118 (1993).

89) Tsukimi Y., Nozue C., Okabe S., J. Gastroenterol. Hepatol., 11, 
$335-340$ (1996)

90) Tsukamoto Y., Tsuchida T., Goto H., Hase S., Arisawa T., Niwa Y., Aliment. Pharmacol. Ther, 7, 581-584 (1993).

91) Szabo S., Hollander D., Am. J. Med., 86, 23-31 (1989).

92) Szabo S., Vattay P., Scarbrough E., Folkman J., Szabo S., Vattay P., Scarbrough E., Folkman J., Am. J. Med., 91, S158-S160 (1991).

93) Okabe S., Takeuchi K., Kunimi H., Kanno M., Kawashima M., Dig. Dis. Sci., 28, 1034-1042 (1983).

94) Okabe S., Jino H., Nishida A., Jpn. J. Pharmacol., 40, 329-337 (1986).

95) Umeda Y., Nakamura K., Jpn. J. Pharmacol., 42, 457-463 (1986).

96) Parmar N. S., Tariq M., Ageel A. M., Prostaglandins Leuk. Med., 24, 255-267 (1986).

97) Konturek S. J., Stachura J., Radecki T., Drozdowicz D., Brzozowski T., Digestion, 38, 103-113 (1987).

98) Penney A. G., Andrews F. J., O'Brien P. E., Dig. Dis. Sci., 39, 934 939 (1994).

99) Arakawa T., Watanabe T., Fukuda T., Yamasaki K., Kobayashi K., Dig. Dis. Sci., 40, 2469-2472 (1995).

100) Konturek S. J., Dembinski A., Warzecha Z., Brzozowski T., Gregory H., Gastroenterology, 94, 1300-1307 (1988).

101) Hase S., Nakazawa S., Tsukamoto Y., Segawa K., Digestion, 42, $135-142$ (1989).

102) Konturek S. J., Brzozowski T., Dembinski A., Warzecha A., Drozdowicz D., Hepatogastroenterology, 35, 25-29 (1998).

103) Itoh M., Imai S., Joh T., Yokoyama Y., Yasue N., Iwai A., Matsusako K., Endoh K., Kawai T., Takeuchi T., J. Clin. Gastroenterol., 12, S187-S191 (1990).

104) Kuwahara Y., Okabe S., Scand. J. Gastroenterol., 162, S162-S165 (1989).

105) Chao J. C., Liu K. Y., Chen S. H., Fang C. L., Tsao C. W., World J Gastroenterol., 9, 2261-2265 (2003).

106) Perez-Aisa A., Sopena F., Arceiz E., Ortego J., Sainz R., Lanas A., Dig. Liver Dis., 35, 397-403 (2003).

107) Mine T., Kushima R., Fujita T., J. Clin. Gastroenterol., 25 (Suppl. 1), S111-S115 (1997).

108) Wingren U., Brown T. H., Watkins B. M., Larson G. M., Scand. J. Gastroenterol., 24, 1102-1106 (1989).

109) Konturek S. J., Brozozowski T., Bielanski W., Warzecha Z., Drozdowicz D., Am. J. Med., 86, 32-37 (1989).

110) Tarnawski A., Stachura J., Durbin T., Sarfeh I. J., Gergely H., Gastroenterology, 102, 695-698 (1992).

111) Fujisawa K., Miyamoto Y., Nagayama M., J. Oral Pathol. Med., 32, $358-366$ (2003).

112) Yabu M., Shinomura Y., Minami T., Matsuzawa Y., Histochemistry, 100, 409-413 (1993).

113) Ernst H., Konturek P. C., Brzozowski T., Konturek S. J., Hahn E. G., J. Physiol. Pharmacol., 47, 443 - 454 (1996).

114) Ernst H., Konturek P., Hahn E. G., Brzozowski T., Konturek S. J., Gut, 39, 172-175 (1996).

115) Konturek S. J., Brzozowski T., Majka J., Szlachcic A., Bielanski W., Stachura J., Otto W., Gut, 34, 881-887 (1993).

116) Akiba Y., Nakamura M., Oda M., Kimura H., Miura S., Tsuchiya M., Ishii H., J. Clin. Gastroenterol., 25, S122-S128 (1997).

117) Nakamura M., Oda M., Inoue J., Ito T., Akiba Y., Kitajima M., Tsuchiya M., Ishii H., Dig. Dis. Sci., 40, 1451-1458 (1995).

118) Brzozowski T., Konturek P. C., Konturek S. J., Schuppan D., Drozdowicz D., Kwiecien S., Majka J., Nakamura T., Hahn E., Digestion, 64, 15-29 (2001).

119) Udagawa A., Shiota G., Ichiba M., Murawaki Y., Scand. J. Gastroenterol., 38,141-146 (2003).

120) Brzozowski T., Konturek P. C., Konturek S. J., Pajdo R., Schuppan D., Drozdowicz D., Ptak A., Pawlik M., Nakamura T., Hahn E. G., J. Physiol. Pharmacol., 51, 751-773 (2000).

121) Arceiz E., Lanas A., Ortego J., Sainz R., Eur. J. Gastroenterol. Hepatol., 9, 1179-1184 (1997).

122) Baatar D., Kawanaka H., Szabo I. L., Pai R., Jones M. K., Kitano S. Tarnawski A. S., Gastroenterology, 122, 458-468 (2002).

123) Machowska A., Szlachcic A., Pawlik M., Brzozowski T., Konturek S J., Pawlik W. W., J. Physiol. Pharmacol., 55, S91-S104 (2004).

124) Gunal O., Oktar B. K., Ozcinar E., Tansuker D., Arbak S., Yegen B. C., Regul. Pept., 106, 81-88 (2002).

125) Reubi J. C., Waser B., Schmassmann A., Halter F., Gastroenterology, 107, 339-346 (1994)
126) Sun F. P., Song Y. G., Cheng W., Zhao T., Yao Y. L., World J. Gastroenterol., 8, 375-378 (2002).

127) Gisbert J. P., Pajares J. M., Aliment. Pharmacol. Ther., 21, 795-804 (2005).

128) Keto Y., Takahashi S., Okabe S., Dig. Dis. Sci., 44, 257-265 (1999).

129) Konturek P. C., Brzozowski T., Kania J., Konturek S. J., Kwiecien S., Pajdo R., Hahn E. G., Eur. J. Pharmacol., 472, 213-220 (2003).

130) Becker J. C., Hertel M., Markmann A., Shahin M., Werner S., Domschke W., Pohle T., Scand. J. Gastroenterol., 38, 260-267 (2003).

131) Watanabe T., Higuchi K., Hamaguchi M., Tanigawa T., Wada R., Tominaga K., Fujiwara Y., Arakawa T., Dig. Dis. Sci., 47, 15821589 (2002).

132) Brzozowski T., Konturek P. C., Brzozowski T., Konturek S. J., Kwiecien S., Pajdo R., Drozdowicz D., Pawlik M., Ptak A., Hahn E. G., J. Pineal. Res., 32, 149-162 (2002).

133) Konturek P. C., Brzozowski T., Sulekova Z., Brzozowska I., Duda A., Meixner H., Hahn E. G., Konturek S. J., Eur. J. Pharmacol., 414, 87-97 (2001).

134) Li Y., Wang W. P., Wang H. Y., Cho C. H., Eur. J. Pharmacol., 399, 205-214 (2000).

135) Kurinets A., Lichtenberger L. M., Dig. Dis. Sci., 43, 786-790 (1998).

136) Motilva V., Martin M. J., Luque M. I., Alarcon de la Lastra C., Gen. Pharmacol., 27, 545-550 (1996).

137) Jones M. K., Kawanaka H., Baatar D., Szabo I. L., Tsugawa K., Pai R., Koh G. Y., Kim I., Sarfeh I. J., Tarnawski A. S., Gastroenterology, 121, 1040-1047 (2001).

138) Chai J., Baatar D., Tarnawski A., Gastroenterology, 126, 1809-1818 (2004).

139) Baatar D., Jones M. K., Tsugawa K., Pai R., Moon W. S., Koh G. Y., Kim I., Kitano S., Tarnawski A. S., Am. J. Pathol., 161, 1449-1457 (2002).

140) Matoba Y., Katayama H., Ohami H., J. Gastroenterol., 31, 777-784 (1996).

141) Majno G., Gabbiani G., Hirschel B. J., Ryan G. B., Statkov P. R., Science, 173, 548-550 (1971).

142) Nakamura M., Oda M., Inoue J., Ito T., Akiba Y., Kitajima M., Tsuchiya M., Ishii H., Dig. Dis. Sci., 40, 2477-2480 (1995).

143) Tsukimi Y., Okabe S., Jpn. J. Pharmacol., 66, 405-412 (1994).

144) Hayashida H., Ishihara K., Ichikawa T., Okayasu I., Kurihara M., Saigenji K., Hotta K., Scand. J. Gastroenterol., 36, 467-473 (2001).

145) Wen C. Y., Ito M., Chen L. D., Matsuu M., Shichijo K., Nakayama T., Nakashima M., Xu Z. M., Ohtsuru A., Hsu C. T., Sekine I., J. Gastroenterol., 38, 431-435 (2003).

146) Wen C. Y., Ito M., Wang H., Chen L. D., Xu Z. M., Matsuu M., Shichijo K., Nakayama T., Nakashima M., Sekine I., World J. Gastroenterol., 9, 788-790 (2003).

147) Wen C. Y., Ito M., Matsuu M., Fukuda E., Shichijo K., Nakashima M., Nakayama T., Sekine I., Life Sci., 70, 2997-3005 (2002).

148) Brzozowski T., Konturek P. C., Konturek S. J., Sliwowski Z., Pajdo R., Drozdowicz D., Ptak A., Hahn E. G., Microsc. Res. Tech., 53, 343-353 (2001).

149) Guo J. S., Cho C. H., Lam Liu E. S., Choy H. T., Wang J. Y., Leung Koo M. W., Toxicol. Appl. Pharmacol., 183, 41-45 (2002).

150) Kobayashi N., Kataoka T., Ono A., Tsukimi Y., Okabe S., J. Physiol. Pharmacol., 52, 195-210 (2001)

151) Shahin M., Konturek J. W., Pohle T., Schuppan D., Herbst H., Domschke W., Microsc. Res. Tech., 53, 396-408 (2001).

152) Tominaga K., Higuchi K., Watanabe T., Fujiwara Y., Kim S., Arakawa T., Iwao H., Kuroki T., Dig. Dis. Sci., 46, 311-317 (2001).

153) Ohmiya N., Saga S., Ohbayashi M., Kozaki K., Miyaishi O., Kobayashi M., Kasuya S., Arisawa T., Goto H., Hayakawa T., Histochem. Cell Biol., 108, 27-34 (1997).

154) Konturek S. J., Brzozowski T., Majka J., Pytko-Polonczyk J., Stachura J., Eur. J. Pharmacol., 239, 215-217 (1993).

155) Brzozowski T., Konturek S. J., Drozdowicz D., Dembinski A., Stachura J., Digestion, 56, 463-471 (1995).

156) Brzozowska I., Konturek P. C., Brzozowski T., Konturek S. J., Kwiecien S., Pajdo R., Drozdowicz D., Pawlik M., Ptak A., Hahn E. G., J. Pineal Res., 32, 149-162 (2002).

157) Guo J. S., Cho C. H., Wang W. P., Shen X. Z., Cheng C. L., Koo M. W., World J. Gastroenterol., 9, 1767-1771 (2003).

158) Tatemichi M., Ogura T., Sakurazawa N., Nagata H., Sugita M., Esumi H., Int. J. Exp. Pathol., 84, 213-220 (2003). 
159) Tramontana M., Renzi D., Calabro A., Panerai C., Milani S., Surrenti C., Evangelista S., Scand. J. Gastroenterol., 29, 406-413 (1994).

160) Konturek P. C., Brzozowski T., Burnat G., Kwiecien S., Pawlik T., Hahn E. G., Konturek S. J., J. Physiol. Pharmacol., 55, 179-192 (2004).

161) Ma L., Chow J. Y., Wong B. C., Cho C. H., Life Sci., 66, PL213-220 (2000).

162) Kang J. Y., Teng C. H., Chen F. C., Gut, 38, 832-836 (1996).

163) Miner P., Aliment. Pharmacol. Ther, 20, S20-S29 (2004).

164) Ligumsky M., Lysy J., Siguencia G., Friedlander Y., J. Clin. Gastroenterol., 33, 32-35 (2001).

165) Ryberg B., Mattsson H., Carlsson E., Digestion, 39, 91—99 (1988).

166) Decktor D. L., Pendleton R. G., Dellner A. T., Davis M. A., J. Pharmacol. Exp. Ther, 249, 1-5 (1989).

167) Olbe L., Cederbberg C., Lind T., Olausson M., Scand. J. Gastroenterol., 24 (Suppl. 166), 27-32 (1989).

168) Ono M., Sato H., Kazumori H., Yuki M., Rumi M. A., Ortega-Cava C. F., Ishihara Y., Ishihara S., Adachi K., Kinoshita Y., J. Lab. Clin. Med., 142, 364-371 (2003).

169) Hammer T. A., Sandvik A. K., Waldum H. L., Scand. J. Gastroenterol., 33, 595-599 (1998).

170) Omura N., Kashiwagi H., Aoki T., Omura K., Fukuchi Y., J. Gastroenterol., 32, 740-746 (1997).

171) Takehara Y., Sumii K., Tari A., Yoshihara M., Sumii M., Haruma K., Kajiyama G., Wu S. V., Walsh J. H., Am. J. Physiol., 271, G799G804 (1996).

172) Chen D., Hakanson R., Sundler F., Cell Tissue Res., 272, 71-77 (1993).
173) Lee H., Hakanson R., Karlsson A., Mattsson H., Sundler F., Digestion, 51, 125-132 (1992).

174) Fukui H., Kinoshita Y., Maekawa T., Okada A., Waki S., Hassan S., Okamoto H., Chiba T., Gastroenterology, 115, 1483-1493 (1998).

175) Miyazaki Y., Shinomura Y., Tsutsui S., Zushi S., Higashimoto Y., Kanayama S., Higashiyama S., Taniguchi N., Matsuzawa Y., Gastroenterology, 116, 78-89 (1999).

176) Ito M., Segami T., Inaguma K., Suzuki Y., Eur. J. Pharmacol., 263, 253-259 (1994).

177) Li H., Helander H. F., Dig. Dis. Sci., 41, $40-48$ (1996).

178) Brzozowska I., Konturek P. C., Brzozowski T., Konturek S. J., Kwiecien S., Pajdo R., Drozdowicz D., Pawlik M., Ptak A., Hahn E. G., J. Pineal Res., 32, 149-162 (2002).

179) Umeda Y., Nakamura K., Jpn. J. Pharmacol., 42, 457-463 (1986).

180) Mizuno H., Sakamoto C., Matsuda K., Wada K., Uchida T., Noguchi H., Akamatsu T., Kasuga M., Gastroenterology, 112, 387-397 (1997).

181) Guo J. S., Cho C. H., Wang J. Y., Koo M. W., Scand. J. Gastroenterol., 37, 17-22 (2002).

182) Tsukimi Y., Nakai H., Itoh S., Amagase K., Okabe S., J. Physiol. Pharmacol., 52, 391-406 (2001).

183) Lamb K., Kang Y. M., Gebhart G. F., Bielefeldt K., Gastroenterology, 125, 1410-1418 (2003).

184) Mei Q., Korsgren M., Erjefalt J. S., Sundler F., Eur. J. Gastroenterol. Hepatol., 14, 503-511 (2002).

185) Naito Y., Yoshikawa T., Matsuyama K., Yagi N., Arai M., Nakamura Y., Nishimura S., Yoshida N., Kondo M., J. Clin. Gastroenterol., 21, S82-S86 (1995). 